C1999 International Monetary Fund

March 1999

IMF Staff Country Report No. 99/17

\title{
Madagascar: Statistical Annex
}

This Statistical Annex report on Madagascar was prepared by a staff team of the International Monetary Fund as background documentation for the periodic consultation with this member country. As such, the views expressed in this document are those of the staff team and do not necessarily reflect the views of the Government of Madagascar or the Executive Board of the IMF.

Copies of this report are available to the public from

International Monetary Fund - Publication Services

700 19th Street, N.W. • Washington, D.C. 20431

Telephone: (202) 623-7430 • Telefax: (202) 623-7201

Telex (RCA): 248331 IMF UR

E-mail: publications@imf.org

Internet: http://www.imf.org

Price: $\$ 15.00$ a copy

\section{International Monetary Fund \\ Washington, D.C.}




\title{
INTERNATIONAL MONETARY FUND
}

\author{
MADAGASCAR
}

\section{Statistical Annex}

Prepared by a staff team consisting of Mrs. Schmitz, Mr. Franks, Mr. Bessaha, Ms. Zephirin, Mr. Fanizza, and Ms. Elborgh-Woytek (all AFR)

Approved by the African Department

January 20, 1999

Contents

Page

Basic Data 3

Social and Demographic Indicators, $1996 \ldots \ldots \ldots \ldots \ldots \ldots \ldots \ldots \ldots$

Tables

1. Growth and Structure of GDP, $1994-98 \ldots \ldots \ldots \ldots \ldots \ldots \ldots \ldots$

2. Availability and Use of Resources at Constant 1984 Prices, 1994-98 _. . . . . . . . 7

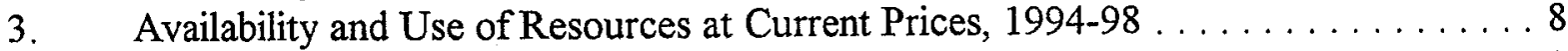

4. Production of Rice and Other Food Crops, 1994-98 . . . . . . . . . . . . . . 9

5. Rice Production, Imports, and Availability, $1961-98 \ldots \ldots \ldots \ldots \ldots \ldots$

6. Retail Prices of Ordinary Rice, $1991-98 \ldots \ldots \ldots \ldots \ldots \ldots \ldots \ldots \ldots \ldots \ldots$

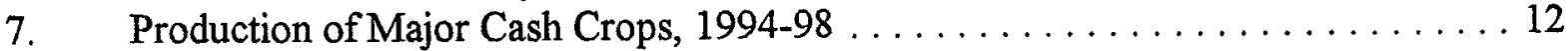

8. Indicative Producer Prices for Major Crops, $1994-97 \ldots \ldots \ldots \ldots \ldots$. . . . . . . 13

9. Index of Industrial Production, $1994-97 \ldots \ldots \ldots \ldots \ldots \ldots \ldots \ldots$

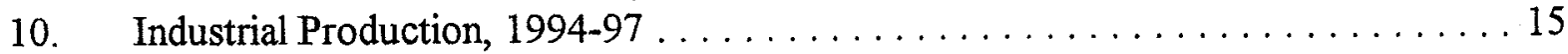

11. Economic Activities of Manufacturing Enterprises in the Export Processing Zones (EPZs), 1994-96 . . . . . . . . . . . . . . . . . . 17

12. Number of Enterprises with Operating Permits under the Export

Processing Zone Regime, $1994-98 \ldots \ldots \ldots \ldots \ldots \ldots \ldots \ldots \ldots$

13. Production and Export of Major Minerals, $1994-98 \ldots \ldots \ldots \ldots \ldots \ldots \ldots$

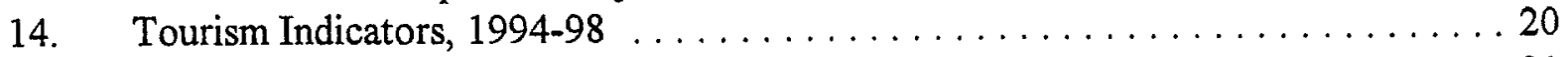

15. Production and Consumption of Electricity, $1994-98 \ldots \ldots \ldots \ldots \ldots \ldots \ldots \ldots 21$

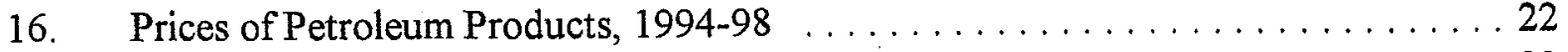

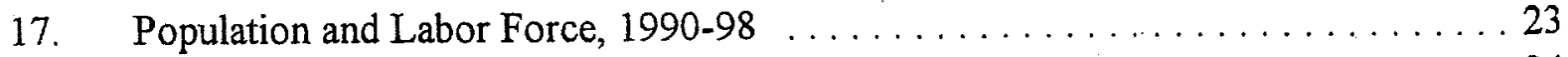

18. Distribution of Population and Civil Servants, $1990-98 \ldots \ldots \ldots \ldots \ldots$ 
19. Minimum and Maximum Monthly Wages, $1984-98 \ldots \ldots \ldots \ldots \ldots$

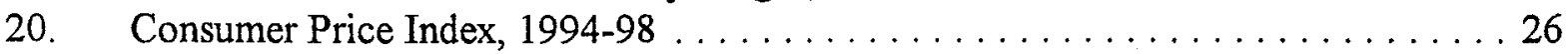

21. Central Government Financial Operations, $1994-98 \ldots \ldots \ldots \ldots \ldots \ldots \ldots \ldots$

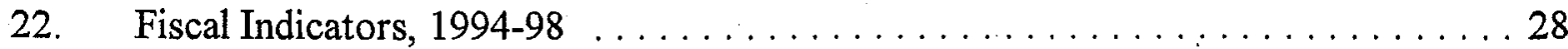

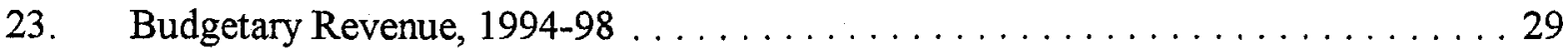

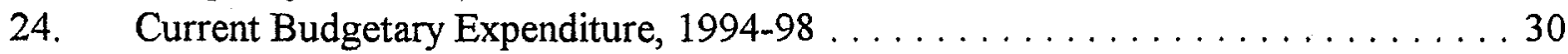

25. Central Government Personnel Expenditure and

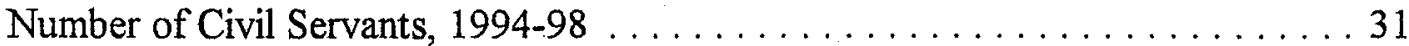

26. Structure and Adjustment of Public Sector Salaries, 1994-98 . . . . . . . . . . 32

27. Central Government Capital Expenditure, $1994-98 \ldots \ldots \ldots \ldots \ldots \ldots$. . . . . . . 33

28. Revenue and Expenditure of the National Social Security Fund, 1994-98 . . . . . 34

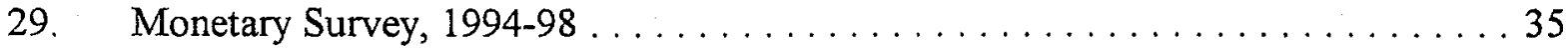

30. Summary Accounts of the Central Bank, $1994-98 \ldots \ldots \ldots \ldots \ldots \ldots$

31. Foreign Reserve Assets and Liabilities of the Central Bank, 1994-98 . . . . . . 37

32. Summary Accounts of the Commercial Banks, 1994-98 . . . . . . . . . . . . 38

33. Structure of Market Interest Rates, $1991-98 \ldots \ldots \ldots \ldots \ldots \ldots \ldots$

34. Structure of Commercial Banks' Interest Rates, 1991-98 . . . . . . . . . . . . . 40

35. Issues and Outstandings of Treasury Bills, $1993-98 \ldots \ldots \ldots \ldots \ldots \ldots \ldots$

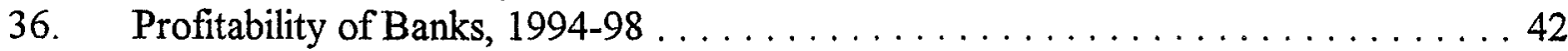

37. Summary Balance of Payments, $1994-98 \ldots \ldots \ldots \ldots \ldots \ldots \ldots \ldots \ldots$

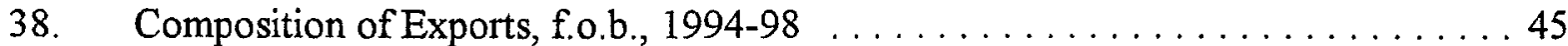

39. Composition of Imports, c.i.f., $1994-98 \ldots \ldots \ldots \ldots \ldots \ldots \ldots \ldots \ldots$

40. Direction of Trade, $1994-98 \ldots \ldots \ldots \ldots \ldots \ldots \ldots \ldots \ldots \ldots \ldots$

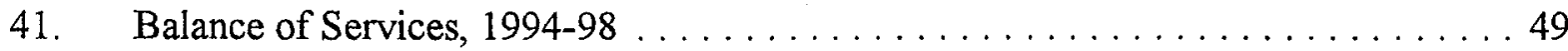

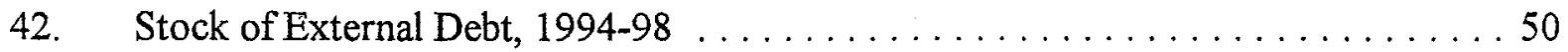

Figures

1. Selected Economic Indicators, $1994-98 \ldots \ldots \ldots \ldots \ldots \ldots \ldots \ldots \ldots$

2. Selected Exchange Rate Indices, January 1990-December $1998 \ldots \ldots \ldots \ldots . . . .52$

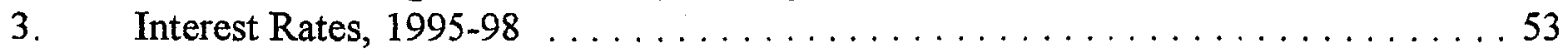

4. Commercial Banks' Interest Rates and Inflation, $1995-98 \ldots \ldots \ldots \ldots \ldots \ldots$

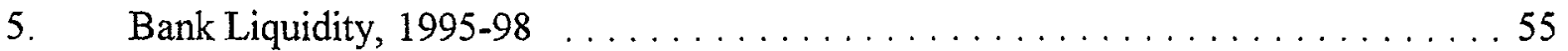


Madagascar: Basic Data

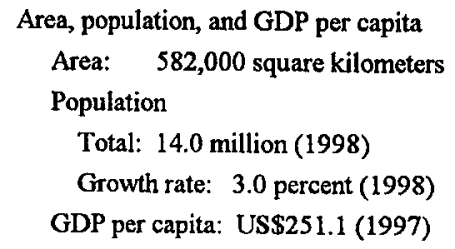

\begin{tabular}{|c|c|c|c|c|c|}
\hline & 1994 & 1995 & 1996 & 1997 & $\begin{array}{r}1998 \\
\text { Est. }\end{array}$ \\
\hline National accounts & \multicolumn{5}{|c|}{ (In billions of Malagasy francs) } \\
\hline GDP at current prices & 9,131 & 13,479 & 16,224 & 18,051 & 20,398 \\
\hline \multirow[t]{2}{*}{ GDP at constant 1984 prices } & 1,899 & 1,931 & 1,973 & 2,043 & 2,123 \\
\hline & \multicolumn{5}{|c|}{ (Annual percentage change at 1984 prices) } \\
\hline Agricultural sector (at factor cost) & -0.5 & 1.9 & 2.5 & 1.9 & 1.5 \\
\hline Industrial sector (at factor cost) & -1.0 & 1.8 & 2.0 & 4.1 & 4.2 \\
\hline \multirow[t]{2}{*}{ Services sector (at factor cost) } & 1.2 & 1.5 & 2.1 & 4.5 & 4.3 \\
\hline & \multicolumn{5}{|c|}{ (In percent of GDP) } \\
\hline Consumption & 96.9 & 97.2 & 93.9 & 96.7 & 94.5 \\
\hline Gross domestic investment & 10.9 & 10.9 & 11.6 & 11.8 & 13,4 \\
\hline Gross domestic savings & 3.1 & 2.8 & 6.1 & 3.3 & 5.5 \\
\hline Current account balance $\mathbf{l}$ & -7.0 & -7.0 & -3.7 & -2.4 & -4.0 \\
\hline Price movements & \multicolumn{5}{|c|}{ (Annual percentage change) } \\
\hline GDP deflator & 41.6 & 45.2 & 17.8 & 7.4 & 8.8 \\
\hline Consumer price index (traditional basket) $2 /$ & 61.2 & 37.3 & 8.3 & 4.8 & 6.3 \\
\hline Government finance & \multicolumn{5}{|c|}{ (In billions of Malagasy francs) } \\
\hline Current revenue and grants & $1,036.0$ & $1,542.0$ & $2,091.0$ & $2,703.0$ & $2,875.0$ \\
\hline Current expenditure & $1,168.0$ & $1,523.0$ & $1,704.0$ & $1,960.0$ & $2,089.0$ \\
\hline Capital expenditure & 636.0 & 850.0 & $1,180.0$ & $1,177.0$ & $1,612.0$ \\
\hline Overall balance on a cash basis (deficit -) & -779.0 & 787.0 & -790.0 & -573.0 & -928.0 \\
\hline Net domestic financing & 213.0 & -38.0 & -15.0 & -119.0 & 879.0 \\
\hline Of which: domestic banks (net) & 201.0 & -43.0 & -45.0 & -146.0 & 726.0 \\
\hline & \multicolumn{5}{|c|}{ (In percent of GDP) } \\
\hline Overall balance on a cash basis (deficit -) & -8.5 & -5.8 & -4.9 & -3.2 & -5.3 \\
\hline \multicolumn{6}{|l|}{ Overall deficit (commitment basis) } \\
\hline Excluding total grants & -11.4 & -9.1 & -9.1 & -7.7 & -7.4 \\
\hline Money and credit & \multicolumn{5}{|c|}{ (In billions of Malagasy francs) } \\
\hline Foreign assets (net) & 373.7 & 616.3 & $1,175.3$ & $1,771.1$ & $1,370.2$ \\
\hline \multicolumn{6}{|l|}{ Domestic credit } \\
\hline Claims on the government (net) & 837.1 & 811.3 & 784.5 & 680.1 & $1,410.3$ \\
\hline Claims on the economy & $1,356.5$ & $1,563.7$ & $1,599.4$ & $1,814.1$ & $1,829.0$ \\
\hline Broad money & $2,339.4$ & $2,717.3$ & $3,209.7$ & $3,846.2$ & $4,265.0$ \\
\hline
\end{tabular}


Madagascar: Basic Data (concluded)

\begin{tabular}{|c|c|c|c|c|c|}
\hline & 1994 & 1995 & 1996 & 1997 & $\begin{array}{r}1998 \\
\text { Est. }\end{array}$ \\
\hline & \multicolumn{5}{|c|}{ (Annual percentage change) } \\
\hline \multicolumn{6}{|l|}{ Domestic credit } \\
\hline Claims on the Government (net) & 25.1 & -3.1 & -3.3 & -13.3 & 107.4 \\
\hline Claims on the economy & 25.6 & 15.3 & 2.3 & 13.4 & 0.8 \\
\hline Broad money & 48.3 & 16.2 & 18.1 & 19.8 & 10.9 \\
\hline Balance of payments & \multicolumn{5}{|c|}{ (In millions of SDRs, unless otherwise indicated) } \\
\hline Exports, f.o.b. & 312.4 & 344.6 & 360.6 & 366.7 & 385.2 \\
\hline Of which: coffee & 59.9 & 61.2 & 42.6 & 23.9 & 31.3 \\
\hline Imports, f.o.b. & 381.0 & 414.0 & 444.0 & 495.6 & 493.9 \\
\hline Trade balance & -68.6 & -69.4 & -83.4 & -128.8 & -108.7 \\
\hline Services (net) & -189.9 & -193.4 & -171.6 & -157.3 & -153.9 \\
\hline Of which: interest payments due & -99.9 & -108.2 & -111.1 & -71.8 & -51.6 \\
\hline Unrequited transfers (net) & 113.1 & 116.5 & 151.7 & 224.4 & 152.2 \\
\hline Private transfers (net) & 32.1 & 49.5 & 60.7 & 84.2 & 60.0 \\
\hline Public transfers (net) $3 /$ & 81.0 & 67.0 & 91.0 & 140.2 & 92.2 \\
\hline \multicolumn{6}{|l|}{ Current account balance } \\
\hline In percent of GDP 4/ & -7.0 & -7.0 & -3.7 & -2.4 & -4.0 \\
\hline Capital account (net) & -69.6 & -37.0 & 26.5 & 81.5 & 22.2 \\
\hline Of which: drawings & 52.3 & 54.9 & 60.0 & 152.3 & 73.7 \\
\hline amortization & -155.5 & -142.8 & -104.9 & -72.6 & -89.9 \\
\hline direct investment & 4.0 & 6.4 & 7.0 & 10.0 & 20.5 \\
\hline Overall balance & -215.1 & -183.3 & -76.8 & 19.7 & -88.2 \\
\hline Debt relief and cancellation & 0.0 & 0.0 & 0.0 & 803.0 & 387.3 \\
\hline IMF (net) & -8.2 & -9.4 & 1.9 & 0.7 & -10.3 \\
\hline Arrears (reduction - ) & 208.4 & 223.1 & 169.3 & -783.2 & -353.1 \\
\hline Reserves (net) (increase -) & 14.9 & -30.4 & -94.5 & -40.2 & 64.3 \\
\hline Gross official reserves 5/ & 3.6 & 5.9 & 12.3 & 14.0 & 9.3 \\
\hline Outstanding external debt 6 / & $2,826.8$ & $2,933.2$ & $3,085.1$ & $3,104.7$ & $2,938.5$ \\
\hline \multicolumn{6}{|l|}{ Exchange rates (period averages) } \\
\hline Malagasy francs per SDR & $4,413.5$ & $6,474.4$ & $5,887.2$ & $7,013.6$ & $7,382.8$ \\
\hline Malagasy franes per U.S. dollar & $3,871.1$ & $3,423.0$ & $4,328.5$ & $5,284.7$ & $5,574.4$ \\
\hline
\end{tabular}

1/ Including official transfers.

2/End of period.

3/ Including project grants.

4/ Including official transfers.

$5 /$ In weeks of imports of goods and services.

6/ After debt relief. 
Madagascar: Social and Demographic Indicators 1996 1/

Land area (square kilometers)

$581,540.0$

Population

Total (in millions) (1998)

14.0

Urban population (percent of total) 27.0

$\begin{array}{ll}\text { Population density (people per sq. } \mathrm{km} \text { ) } & 23.6\end{array}$

Population density, rural (people per sq. km) (1995) 2/ 379.4

$\begin{array}{ll}\text { Population growth (annual percentage) } & 3.0\end{array}$

Life expectancy at birth (years)

Overall

58.1

Women

59.6

Men

56.6

Crude birth rate (per 1,000)

40.9

Crude death rate (per 1,000)

10.8

Infant mortality rate (per 1,000$)$

87.7

Education

Illiteracy rate, adult total (percentage of people over 15) (1990)

19.8

Primary education, pupils (in thousands) (1994)

Secondary education, general pupils (in thousands) (1992)

304.8

Secondary education, vocational pupils (in thousands) (1992)

Primary school enrollment (percentage of relevant age group) (1993)

72.0

Secondary school enrollment (percentage of relevant age group) (1993)

14.0

Tertiary school enrollment (percentage of relevant age group) (1993)

Health

$\begin{array}{ll}\text { Hospital beds (per 1,000) (1990) } & 0.9\end{array}$

$\begin{array}{ll}\text { Physicians (per 1,000) (1990) } & 0.1\end{array}$

Safe water (percentage of population with access) (1994) 29.0

Sanitation (percentage of population with access) (1994) 15.0

Child immunization (under 12 months, percent)

DPT

67.0

Measles

59.0

Sources: Malagasy authorities; and World Bank, World Development Indicators .

1/ Most recent estimates available.

2/ Rural population density is the rural population divided by the arable land area. 
Table 1. Madagascar: Growth and Structure of GDP, 1994-98

\begin{tabular}{|c|c|c|c|c|c|}
\hline & 1994 & 1995 & 1996 & 1997 & $\begin{array}{r}1998 \\
\text { Est. }\end{array}$ \\
\hline & \multicolumn{5}{|c|}{ (In billions of Malagasy francs at 1984 prices) } \\
\hline Agriculture & 655.0 & 667.3 & 684.0 & 696.9 & 707.4 \\
\hline Industry & 221.5 & 225.4 & 229.8 & 239.3 & 249.4 \\
\hline Services & 892.7 & 906.4 & 925.6 & 967.4 & $1,009.0$ \\
\hline Of which: public sector $1 /$ & 108.1 & 105.7 & 108.1 & 108.1 & $\ldots$ \\
\hline Imputed charges & -31.5 & -32.0 & -32.5 & -33.1 & -37.1 \\
\hline GDP at factor cost & $1,737.7$ & $1,767.1$ & $1,806.9$ & $1,870.5$ & $1,928.6$ \\
\hline Indirect taxes & 161.6 & 164.1 & 165.8 & 172.5 & 193.9 \\
\hline \multirow[t]{2}{*}{ GDP at market prices } & $1,899.3$ & $1,931.2$ & $1,972.7$ & $2,043.0$ & $2,122.5$ \\
\hline & \multicolumn{5}{|c|}{ (Annual change in percent at 1984 prices) } \\
\hline Agriculture & -0.5 & 1.9 & 2.5 & 1.9 & 1.5 \\
\hline Industry & -1.0 & 1.8 & 2.0 & 4.1 & 4.2 \\
\hline Services & 1.2 & 1.5 & 2.1 & 4.5 & 4.3 \\
\hline Of which: public sector $1 /$ & -0.9 & -2.2 & 2.3 & $\ldots$ & $\ldots$ \\
\hline GDP at market prices & 0.0 & 1.7 & 2.1 & 3.6 & 3.9 \\
\hline Real GDP per capita & -2.8 & -2.4 & -0.9 & 0.5 & 0.9 \\
\hline \multirow[t]{2}{*}{ GDP deflator } & 41.6 & 45.2 & 17.8 & 7.4 & 8.8 \\
\hline & \multicolumn{5}{|c|}{ (In percent of GDP at current market prices) } \\
\hline Agricutture & 36.6 & 30.5 & 29.6 & 29.2 & 28.0 \\
\hline Industry & 12.7 & 12.7 & 12.6 & 12.4 & 12.5 \\
\hline Services & 46.0 & 51.1 & 52.1 & 52.0 & 52.3 \\
\hline Of which: public sector $1 /$ & 4.6 & 4.3 & 4.2 & 4.6 & 0.0 \\
\hline Imputed charges & -1.4 & -1.4 & -1.2 & -1.2 & -1.2 \\
\hline GDP at factor cost & 93.9 & 92.9 & 93.1 & 92.5 & 91.7 \\
\hline Indirect taxes & 6.1 & 7.1 & 6.9 & 7.5 & 8.3 \\
\hline \multicolumn{6}{|l|}{ Net imports of goods and } \\
\hline nonfactor services & 5.9 & 4.9 & 3.2 & 5.0 & 5.6 \\
\hline Total available resources & 105.9 & 104.9 & 103.2 & 105.0 & 105.6 \\
\hline Consumption & 95.0 & 94.0 & 91.6 & 93.1 & 92.3 \\
\hline Of which: public sector $1 /$ & 6.9 & 6.7 & 6.1 & 7.6 & 6.1 \\
\hline Gross investment & 10.9 & 10.9 & 11.6 & 11.9 & 13.3 \\
\hline Of which: public sector $1 /$ & 6.4 & 6.0 & 6.7 & 6.3 & 7.2 \\
\hline Gross domestic savings & 5.0 & 6.0 & 8.4 & 6.9 & 7.7 \\
\hline
\end{tabular}

Sources: Ministry of Finance and Economy; Ministry of Budget and Development of Autonomous Provinces; and Fund staff estimates.

1/ Excluding public enterprises. 
Table 2. Madagascar: Availability and Use of Resources at Constant 1984 Prices, 1994-98 (In billions of Malagasy francs at 1984 prices)

\begin{tabular}{|c|c|c|c|c|c|}
\hline & 1994 & 1995 & 1996 & 1997 & $\begin{array}{r}1998 \\
\text { Est. }\end{array}$ \\
\hline Agriculture & 655.0 & 667.3 & 684.0 & 696.9 & 707.4 \\
\hline Industry & 221.5 & 225.4 & 229.8 & 239.3 & 249.4 \\
\hline Services & 892.7 & 906.4 & 925.6 & 967.4 & $1,009.0$ \\
\hline Of which: public sector $1 /$ & 108.1 & 105.7 & 108.1 & 108.1 & \\
\hline Imputed charges & -31.5 & -32.0 & -32.5 & -33.1 & -37.1 \\
\hline GDP at factor cost & $1,737.7$ & $1,767.1$ & $1,806.9$ & $1,870.5$ & $1,928.6$ \\
\hline Indirect taxes & 161.6 & 164.1 & 165.8 & 172.5 & 193.9 \\
\hline GDP at market prices & $1,899.3$ & $1,931.2$ & $1,972.7$ & $2,043.0$ & $2,122.5$ \\
\hline Net imports of goods and nonfactor services & -6.5 & -9.3 & -11.5 & 28.3 & 48.3 \\
\hline Imports of goods and nonfactor services & 319.2 & 327.2 & 340.0 & 366.6 & 407.5 \\
\hline Exports of goods and nonfactor services & 325.7 & 336.5 & 351.5 & 338.3 & 359.3 \\
\hline Total available resources & $1,892.8$ & $1,921.9$ & $1,961.2$ & $2,071.3$ & $2,170.8$ \\
\hline Consumption & $1,701.0$ & $1,727.1$ & $1,742.1$ & $1,855.5$ & $1,921.6$ \\
\hline Of which: public sector $1 /$ & 153.6 & 157.8 & 151.8 & 174.3 & 137.9 \\
\hline Gross investment & 191.8 & 194.8 & 219.1 & 215.8 & 249.2 \\
\hline \multicolumn{6}{|l|}{ Memorandum items: } \\
\hline GDP deflator $(1984=100)$ & 480.8 & 697.9 & 822.4 & 883.5 & 961.0 \\
\hline Percentage change & 41.6 & 45.2 & 17.8 & 7.4 & 8.8 \\
\hline Real GDP per capita 2/ & 148.7 & 145.2 & 143.9 & 144.7 & 145.9 \\
\hline Percentage change & -2.8 & -2.4 & -0.9 & 0.5 & 0.9 \\
\hline
\end{tabular}

Sources: Ministry of Finance and Economy; Ministry of Budget and Development of Autonomous Provinces; and Fund staff estimates.

1/ Excluding public enterprises.

2/ In thousands of Malagasy francs at 1984 prices. 
Table 3. Madagascar: Availability and Use of Resources at Current Prices, 1994-98

(In billions of Malagasy francs)

\begin{tabular}{|c|c|c|c|c|c|}
\hline & 1994 & 1995 & 1996 & 1997 & $\begin{array}{c}1998 \\
\text { Est. }\end{array}$ \\
\hline Agriculture & $3,342.6$ & $4,109.3$ & $4,807.5$ & $5,266.2$ & $5,719.4$ \\
\hline Industry & $1,155.1$ & $1,716.9$ & $2,046.6$ & $2,244.5$ & $2,549.3$ \\
\hline Services & $4,202.5$ & $6,885.3$ & $8,449.5$ & $9,389.3$ & $10,674.4$ \\
\hline Of which: public sector $1 /$ & 420.7 & 573.7 & 678.6 & 837.2 & $\ldots$ \\
\hline Imputed charges & -129.3 & -186.0 & -195.4 & -207.8 & -237.6 \\
\hline GDP at factor cost & $8,570.9$ & $12,525.5$ & $15,108.2$ & $16,692.2$ & $18,705.5$ \\
\hline Indirect taxes & 560.3 & 953.2 & $1,116.2$ & $1,358.6$ & $1,692.3$ \\
\hline GDP at market prices & $9,131.2$ & $13,478.7$ & $16,224.4$ & $18,050.8$ & $20,397.8$ \\
\hline Net imports of goods and nonfactor services & 538.8 & 666.2 & 525.2 & 894.5 & $1,146.2$ \\
\hline Imports of goods and nonfactor services & $2,720.9$ & $4,282.7$ & $4,219.1$ & $5,476.8$ & $5,946.3$ \\
\hline Exports of goods and nonfactor services & $2,182.1$ & $3,616.5$ & $3,693.9$ & $4,582.3$ & $4,800.1$ \\
\hline Total available resources & $9,670.0$ & $14,144.9$ & $16,749.6$ & $18,945.3$ & $21,543.9$ \\
\hline Consumption & $8,674.4$ & $12,670.0$ & $14,861.6$ & $16,805.8$ & $18,827.6$ \\
\hline Of which: public sector $1 /$ & 628.8 & 904.2 & 985.7 & $1,378.1$ & $1,241.0$ \\
\hline Gross investment & 995.6 & $1,474.9$ & $1,888.0$ & $2,139.5$ & $2,716.3$ \\
\hline Fixed & 995.6 & $1,474.9$ & $1,888.0$ & $2,139.5$ & $2,716.3$ \\
\hline Private & 411.2 & 666.2 & 806.2 & $1,003.3$ & $1,246.3$ \\
\hline Public sector 1/ & 584.4 & 808.7 & $1,081.8$ & $1,136.2$ & $1,470.0$ \\
\hline Gross domestic savings & 456.8 & 808.7 & $1,362.8$ & $1,245.0$ & $1,570.2$ \\
\hline Public sector $1 /$ & 51.2 & 249.8 & 329.5 & 290.1 & 126.8 \\
\hline Private & 405.6 & 558.9 & $1,033.3$ & 954.9 & $1,443.4$ \\
\hline
\end{tabular}

Sources: Ministry of Finance and Economy; Ministry of Budget and Development of Autonomous Provinces; and Fund staff estimates. 
Table 4. Madagascar: Production of Rice and Other Food Crops, 1994-98

(In thousands of tons)

\begin{tabular}{lccccc}
\hline & 1994 & 1995 & 1996 & 1997 & $\begin{array}{r}1998 \\
\text { Prel. }\end{array}$ \\
& & & & & \\
& 2,357 & 2,450 & 2,500 & 2,558 & 2,447 \\
Paddy & 155 & 177 & 180 & 178 & 152 \\
Maize & 2,360 & 2,400 & 2,353 & 2,430 & 2,404 \\
Manioc & 560 & 450 & 500 & 510 & 510 \\
Sweet potatoes & 270 & 275 & 280 & 280 & 280 \\
Potatoes & & & & & \\
\hline
\end{tabular}

Source: Ministry of Agriculture. 
Table 5. Madagascar: Rice Production, Imports, and Availability, 1961-98

\begin{tabular}{|c|c|c|c|c|c|c|}
\hline & \multirow{2}{*}{$\begin{array}{l}\text { Population 1/ } \\
\text { (Thousands) }\end{array}$} & $\begin{array}{c}\text { Production } \\
\text { of Paddy }\end{array}$ & $\begin{array}{c}\begin{array}{c}\text { End-of-year } \\
\text { Stocks }\end{array} \\
\end{array}$ & $\begin{array}{c}\text { Net } \\
\text { Imports } 2 / \\
\end{array}$ & $\begin{array}{r}\text { Total Rice } \\
\text { Available } 3 / \\
\end{array}$ & \multirow{2}{*}{ 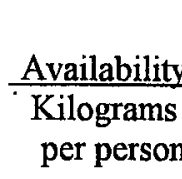 } \\
\hline & & \multicolumn{4}{|c|}{ (In thousands of metric tons) } & \\
\hline 1961 & 5,616 & 1,263 & $\ldots$ & -13 & 672 & 120 \\
\hline 1962 & 5,729 & 1,330 & $\ldots$ & -25 & 697 & 122 \\
\hline 1963 & 5,842 & 1,377 & $\ldots$ & -20 & 727 & 124 \\
\hline 1964 & 5,959 & 1,520 & $\ldots$ & -21 & 805 & 135 \\
\hline 1965 & 6,078 & 1,445 & $\ldots$ & 60 & 844 & 139 \\
\hline 1966 & 6,200 & 1,603 & $\ldots$ & -5 & 864 & 139 \\
\hline 1967 & 6,342 & 1,706 & $\ldots$ & -36 & 891 & 140 \\
\hline 1968 & 6,487 & 1,873 & $\ldots$ & -64 & 952 & 147 \\
\hline 1969 & 6,636 & 1,858 & $\cdots$ & -9 & 999 & 151 \\
\hline 1970 & 6,788 & 1,865 & $\ldots$ & -48 & 965 & 142 \\
\hline 1971 & 6,943 & 1,873 & $\ldots$ & 25 & 1,042 & 150 \\
\hline 1972 & 7,102 & 1,687 & $\ldots$ & 23 & 938 & 132 \\
\hline 1973 & 7,265 & 1,730 & $\ldots$ & 90 & 1,028 & 142 \\
\hline 1974 & 7,431 & 1,844 & 5 & 122 & 1,118 & 150 \\
\hline 1975 & 7,604 & 1,972 & 0 & 59 & 1,135 & 149 \\
\hline 1976 & 7,817 & 2,043 & 5 & 68 & 1,171 & 150 \\
\hline 1977 & 8,035 & 2,067 & 9 & 93 & 1,211 & 151 \\
\hline 1978 & 8,261 & 1,922 & 7 & 152 & 1,196 & 145 \\
\hline 1979 & 8,492 & 2,045 & 0 & 155 & 1,272 & 150 \\
\hline 1980 & 8,730 & 2,109 & 2 & 176 & 1,318 & 151 \\
\hline 1981 & 8,953 & 2,012 & 8 & 193 & 1,278 & 143 \\
\hline 1982 & 9,225 . & 1,970 & 138 & 351 & 1,290 & 140 \\
\hline 1983 & 9,484 & 2,147 & 122 & 185 & 1,365 & 144 \\
\hline 1984 & 9,715 & 2,131 & 47 & 111 & 1,343 & 138 \\
\hline 1985 & 9,984 & 2,060 & 0 & 107 & 1,409 & 141 \\
\hline 1986 & 10,108 & 2,116 & 28 & 162 & 1,424 & 141 \\
\hline 1987 & 10,368 & 2,178 & 37 & 94 & 1,433 & 138 \\
\hline 1988 & 10,635 & 2,149 & 13 & 37 & 1,385 & 130 \\
\hline 1989 & 10,909 & 2,380 & 28 & 89 & 1,547 & 142 \\
\hline 1990 & 11,197 & 2,420 & 28 & 76 & 1,574 & 141 \\
\hline 1991 & 11,783 & 2,342 & 30 & 39 & 1,525 & 133 \\
\hline 1992 & 11,986 & 2,450 & 38 & 59 & 1,568 & 130 \\
\hline 1993 & 12,200 & 2,550 & 40 & 5 & 1,581 & 127 \\
\hline 1994 & 12,542 & 2,357 & 53 & 156 & 1,602 & 126 \\
\hline 1995 & 12,893 & 2,450 & 62 & 152 & 1,559 & 121 \\
\hline 1996 & 13,254 & 2,500 & 56 & 32 & 1,585 & 113 \\
\hline 1997 & 13,625 & 2,558 & $\ldots$ & 56 & 1,663 & 120 \\
\hline $19984 /$ & 14,007 & 2,447 & $\ldots$ & 111 & 1,619 & 114 \\
\hline
\end{tabular}

Source: Ministry of Agriculture.

1/ May differ from data provided by other sources.

2/ Madagascar was a net exporter of rice until 1970 (except for 1965).

3/ Domestic paddy production (less nonhuman consumption) converted to rice equivalent plus net change in stocks and net imports.

4/ Provisional data. 
Table 6. Madagascar: Retail Prices of Ordinary Rice, 1991-98

(In Malagasy francs per kilogram)

\begin{tabular}{|c|c|c|c|c|}
\hline & & $\begin{array}{c}\text { Free } \\
\text { Market }\end{array}$ & $\begin{array}{l}\text { Security } \\
\text { Stocks 1/ }\end{array}$ & $\begin{array}{c}\text { Official } \\
\text { Distribution }\end{array}$ \\
\hline \multicolumn{5}{|l|}{1991} \\
\hline & March & 624 & 612 & \\
\hline & June & 549 & 534 & 525 \\
\hline & September & 665 & 653 & $\ldots$ \\
\hline & December & 893 & 794 & 700 \\
\hline \multicolumn{5}{|l|}{1992} \\
\hline & March & 980 & 969 & $\ldots$ \\
\hline & June & 830 & 689 & 932 \\
\hline & September & 805 & 797 & 950 \\
\hline & December & 860 & 875 & 975 \\
\hline \multicolumn{5}{|l|}{1993} \\
\hline & March & 823 & 787 & 875 \\
\hline & June & 630 & 650 & 800 \\
\hline & September & 770 & 759 & 900 \\
\hline & December & 847 & 861 & 1,000 \\
\hline \multicolumn{5}{|l|}{1994} \\
\hline & March & 1,575 & 1,428 & 1,000 \\
\hline & June & 958 & 914 & 1,000 \\
\hline & September & 1,308 & 1,385 & 850 \\
\hline & December & 1,646 & 1,564 & 1,200 \\
\hline \multicolumn{5}{|l|}{1995} \\
\hline & March & 1,735 & 1,729 & 1,200 \\
\hline & June & 1,555 & 1,506 & 1,800 \\
\hline & September & 1,875 & 1,822 & 1,950 \\
\hline & December & 2,076 & 1,975 & 1,950 \\
\hline \multicolumn{5}{|l|}{1996} \\
\hline & March & 2,168 & 2,066 & 2,150 \\
\hline & June & 1,609 & 1,656 & 2,200 \\
\hline & September & 1,785 & 1,744 & 2,200 \\
\hline & December & 2,055 & 1,919 & 2,250 \\
\hline \multicolumn{5}{|l|}{1997} \\
\hline & March & 2,128 & 2,056 & 2,150 \\
\hline & June & 1,608 & 1,619 & 1,950 \\
\hline & September & 1,876 & 1,906 & 2,036 \\
\hline & December & 2,089 & 2,006 & 2,200 \\
\hline \multicolumn{5}{|l|}{1998} \\
\hline & March & 2,125 & 2,071 & 2,167 \\
\hline & June & 1,875 & 2,000 & 2,172 \\
\hline & September & 1,979 & 1,992 & 2,225 \\
\hline & December $2 /$ & 2,200 & 2,100 & 2,300 \\
\hline
\end{tabular}

Source: Ministry of Commerce and Consumption.

1/ The data refer to the price of rice sold from the food security stock, or stock tampon.

2/ Provisional data. 
Table 7. Madagascar: Production of Major Cash Crops, 1994-98 1/

(In thousands of tons)

\begin{tabular}{|c|c|c|c|c|c|c|}
\hline & & 1994 & 1995 & 1996 & 1997 & $\begin{array}{r}1998 \\
\text { Est. }\end{array}$ \\
\hline \multicolumn{7}{|l|}{ Export crops } \\
\hline \multirow[t]{2}{*}{ Coffee 2/ } & Production & 70.0 & 68.0 & 68.0 & 55.0 & 60.0 \\
\hline & Marketed & 48.0 & 60.0 & 60.0 & 52.0 & 53.0 \\
\hline \multirow[t]{2}{*}{ Vanilla } & Production 3/ & 4.0 & 4.3 & 4.4 & 4.8 & 5.0 \\
\hline & Marketed 4/ & 0.6 & 0.6 & 1.2 & 1.1 & 1.1 \\
\hline \multirow[t]{2}{*}{ Cloves } & Production & 14.0 & 13.0 & 13.0 & 15.5 & 16.5 \\
\hline & Marketed & 13.3 & 13.0 & 12.5 & 15.0 & 15.0 \\
\hline \multirow[t]{2}{*}{ Pepper } & Production & 2.3 & 2.4 & 2.1 & 1.5 & 1.7 \\
\hline & Marketed & 2.3 & 1.8 & 1.9 & 1.5 & 1.7 \\
\hline \multirow[t]{2}{*}{ Cocoa } & Production & 3.0 & 4.0 & 4.8 & 4.3 & 4.3 \\
\hline & Marketed & 2.3 & 3.3 & 4.5 & 4.3 & 4.3 \\
\hline \multirow[t]{2}{*}{ Butter beans } & Production & 5.0 & 7.8 & 7.8 & 8.0 & 7.8 \\
\hline & Marketed & 4.0 & 4.4 & 4.5 & 7.8 & 7.0 \\
\hline Sisal & Production 5/ & 17.2 & 16.3 & 17.0 & 18.0 & 18.0 \\
\hline \multicolumn{7}{|l|}{ Industrial crops } \\
\hline Cotton $6 /$ & Production 5/ & 27.0 & 24.3 & 26.0 & 36.2 & 38.5 \\
\hline \multirow[t]{2}{*}{ Sugarcane } & Production & 2,166 & 2,100 & 2,150 & 2,160 & 2,180 \\
\hline & Marketed & 1,652 & 1,822 & 2,016 & 1,500 & 1,500 \\
\hline \multirow[t]{2}{*}{ Groundnuts } & Production & 28.0 & 30.0 & 36.2 & 35.8 & 34.2 \\
\hline & Marketed & 20.0 & 21.4 & 22.4 & 29.0 & 29.0 \\
\hline
\end{tabular}

Source: Ministry of Agriculture.

1/ Data on total production are approximate; those on marketed production are more accurate.

2/ Unroasted coffee.

3/ Green vanilla.

4/ Prepared vanilla ( $4.6 \mathrm{~kg}$. green $=1 \mathrm{~kg}$. prepared).

$5 /$ Most of the production is marketed.

$6 /$ Seed cotton. 
Table 8. Madagascar: Indicative Producer Prices for Major Crops, 1994-97

(In Malagasy francs per kilogram)

\begin{tabular}{|c|c|c|c|c|c|}
\hline & $\begin{array}{l}\text { Type of } \\
\text { Price }\end{array}$ & 1994 & 1995 & 1996 & 1997 \\
\hline Paddy & Indicative & 648 & 725 & 726 & 726 \\
\hline Coffee & Indicative & 8,200 & 6,000 & 4,570 & $3,000-5,000$ \\
\hline Cloves & Indicative & 1,800 & 1,850 & 1,900 & 3,000 \\
\hline Vanilla & Fixed & 8,450 & 10,000 & 5,150 & $5,000-10,000$ \\
\hline Sugarcane & Indicative & 45 & 75 & 95 & n.a. \\
\hline Pepper & Indicative & 2,500 & 2,700 & 2,850 & n.a. \\
\hline Wheat & Indicative & 850 & $\ldots$ & $\ldots$ & n.a. \\
\hline Soybeans & Indicative & 510 & $\ldots$ & $\ldots$ & n.a. \\
\hline Cotton & Indicative & 964 & 1,615 & 1,934 & n.a. \\
\hline
\end{tabular}

Source: Ministry of Agriculture. 
Table 9. Madagascar: Index of Industrial Production, 1994-97

\begin{tabular}{|c|c|c|c|c|c|}
\hline & Weights & 1994 & 1995 & 1996 & 1997 \\
\hline & \multicolumn{5}{|c|}{$(1984=100)$} \\
\hline Agro-industry and food industries & 29.18 & 111.4 & 114.5 & 116.1 & 111.6 \\
\hline Beverages and tobacco & 7.59 & 96.9 & 105.9 & 129.7 & 142.2 \\
\hline Textiles and clothing & 14.63 & 77.0 & 78.4 & 67.3 & 71.5 \\
\hline Wood industries & 1.09 & 100.6 & 104.0 & 109.6 & 109.9 \\
\hline Paper and printing & 4.68 & 119.3 & 113.3 & 65.9 & 58.6 \\
\hline Leather goods & 2.65 & 76.2 & 61.2 & 59.4 & 25.6 \\
\hline Electrical goods & 2.19 & 90.1 & 102.1 & 95.6 & 102.8 \\
\hline Metal works & 2.43 & 109.1 & 143.0 & 141.7 & 151.4 \\
\hline Chemical industries & 9.05 & 134.8 & 112.2 & 115.9 & 120.2 \\
\hline Energy & 18.27 & 322.2 & 363.7 & 394.2 & 462.2 \\
\hline Construction materials & 2.19 & 147.6 & 163.7 & 165.2 & 190 \\
\hline Transport materials & 1.23 & 95.8 & 90.0 & 92.7 & 96.4 \\
\hline Extractive industries & 3.86 & 102.7 & 118.4 & 110.6 & 120.0 \\
\hline Other & 0.96 & $\ldots$ & $\ldots$ & $\ldots$ & $\ldots$ \\
\hline \multirow[t]{2}{*}{ Total } & 100.0 & 143.2 & 152.5 & 156.5 & 169.7 \\
\hline & \multicolumn{5}{|c|}{ (Annual change in percent) } \\
\hline Agro-industry and food industries & & 3.4 & 2.8 & 1.4 & -3.9 \\
\hline Beverages and tobacco & & 3.4 & 9.3 & 22.5 & 9.6 \\
\hline Textiles and clothing & & 1.3 & 1.8 & -14.2 & 6.2 \\
\hline Wood industries & & 9.5 & 3.4 & 5.4 & 0.3 \\
\hline Paper and printing & & 33.0 & -5.0 & -41.8 & -11.1 \\
\hline Leather goods & & 1.7 & -19.7 & -2.9 & -56.9 \\
\hline Electrical goods & & -4.3 & 13.3 & -6.4 & 7.5 \\
\hline Metal works & & 16.6 & 31.1 & -0.9 & 6.8 \\
\hline Chemical industries & & -5.6 & -16.8 & 3.3 & 3.7 \\
\hline Energy & & -21.9 & 12.9 & 8.4 & 17.3 \\
\hline Construction materials & & 33.1 & 10.9 & 0.9 & 15.0 \\
\hline Transport materials & & 33.1 & -6.1 & 3.0 & 4.0 \\
\hline Extractive industries & & -4.1 & 15.3 & -6.6 & 8.5 \\
\hline Total & & -8.7 & 6.5 & 2.6 & 8.4 \\
\hline
\end{tabular}

Sources: Ministry of Industrialization and Handicrafts; and Ministry of Energy and Mining. 
Table 10. Madagascar: Industrial Production, 1994-97

\begin{tabular}{|c|c|c|c|c|c|}
\hline & Unit & 1994 & 1995 & 1996 & 1997 \\
\hline \multicolumn{6}{|l|}{ Extractive industries } \\
\hline Graphite & Ton & 14,659 & 16,119 & 11,295 & 14,000 \\
\hline Mica & Ton & 563 & 432 & 317 & 500 \\
\hline Chromite & Ton & 75,000 & 105,747 & 139,272 & 140,000 \\
\hline \multicolumn{6}{|l|}{ Food industries } \\
\hline Tapioca & Ton & 53 & 70 & 75 & $\ldots$ \\
\hline Potato flour & Ton & 300 & 210 & 400 & 354 \\
\hline Cattle (slaughtered industrially) & Head & 20,000 & 22,500 & 25,000 & $\ldots$ \\
\hline Hogs (slaughtered industrially) & Head & 1,300 & 1,550 & 1,800 & $\cdots$ \\
\hline Canned meat & Ton & 60 & 59 & 42 & $\cdots$ \\
\hline Cooked pork meats & Ton & 105 & 113 & 137 & $\ldots$ \\
\hline Sugar & Ton & 79,280 & 90,052 & 87,608 & 82,343 \\
\hline Edible oils & Ton & 840 & 751 & 2,000 & 1,900 \\
\hline Beer & Hectoliters & 218,976 & 318,842 & 347,795 & 457,914 \\
\hline Condensed milk & Ton & 2,148 & 3,200 & 2,229 & $\ldots$ \\
\hline Salt & Ton & 75,783 & 70,560 & 41,750 & 46,702 \\
\hline \multicolumn{6}{|l|}{ Tobacco } \\
\hline Chewing tobacco & Ton & 336 & 424 & 813 & 861 \\
\hline Smoking tobacco & Ton & 6 & 7 & 6 & 6 \\
\hline Cigarettes & Ton & 2,280 & 1,969 & 3,371 & 3,159 \\
\hline \multicolumn{6}{|l|}{ Textiles } \\
\hline Cotton cloth & 1,000 meters & 35,042 & 24,257 & 11,052 & 11,572 \\
\hline Bags for packaging & Ton & 1,195 & 950 & 825 & 907 \\
\hline Blankets & Ton & 2,376 & 1,429 & 1,505 & 1,289 \\
\hline String & Ton & 1,366 & 1,286 & 1,144 & 915 \\
\hline \multicolumn{6}{|l|}{ Paper } \\
\hline Rag paper & Ton & 6,611 & 6,940 & 3,963 & 4,646 \\
\hline Finished paper & Ton & 4,505 & 3,690 & 2,208 & 969 \\
\hline
\end{tabular}


Table 10 (concluded). Madagascar: Industrial Production, 1994-97

\begin{tabular}{|c|c|c|c|c|c|}
\hline & Unit & 1994 & 1995 & 1996 & 1997 \\
\hline \multicolumn{6}{|l|}{ Chemical industries } \\
\hline Soap & Ton & 16,837 & 16,654 & 16,500 & 15,071 \\
\hline Accumulators & units & 6,629 & 4,920 & 3,736 & 3,848 \\
\hline Candles & Ton & 1,100 & 2,491 & 584 & 454 \\
\hline Paint & Ton & 2,600 & 1,855 & 2,350 & 2,397 \\
\hline Batteries & 1,000 units & 20,382 & 23,958 & 22,715 & 24,474 \\
\hline Oxygen & Cubic meters & 477,441 & 418,514 & 509,859 & 524,852 \\
\hline Acetylene & Cubic meters & 119,476 & 107,281 & 110,813 & 116,524 \\
\hline \multicolumn{6}{|l|}{ Petroleum refining } \\
\hline Butane & Cubic meters & 2,306 & 3,901 & 5,591 & 9,424 \\
\hline Gasoline & Cubic meters & 41,205 & 64,941 & 70,621 & 96,573 \\
\hline Kerosene & Cubic meters & 36,233 & 48,779 & 54,393 & 72,148 \\
\hline Gas oil & Cubic meters & 56,359 & 111,070 & 93,689 & 125,317 \\
\hline Fuel oil & Cubic meters & 87,528 & 204,533 & 159,500 & 211,522 \\
\hline \multicolumn{6}{|l|}{ Construction materials } \\
\hline Cement & Ton & 8,524 & 38,000 & 38,349 & 44,102 \\
\hline \multicolumn{6}{|l|}{ Metal works } \\
\hline Sheet metal & Ton & 3,361 & 5,492 & 4,711 & 5,031 \\
\hline Trunks and boxes & Ton & 1,500 & 1,259 & 1,441 & 1,499 \\
\hline \multicolumn{6}{|l|}{ Shoes } \\
\hline Leather & 1,000 pairs & 180 & 132 & 158 & 162 \\
\hline Plastic & 1,000 pairs & 659 & 547 & 409 & 339 \\
\hline Other & 1,000 pairs & 150 & 120 & 324 & 330 \\
\hline \multicolumn{6}{|l|}{ Electricity } \\
\hline Hydraulic & 1,000 kilowatt-hours & 389,200 & 415,000 & 442,430 & 488,718 \\
\hline Thermal & 1,000 kilowatt-hours & 156,800 & 137,000 & 120,790 & 127,593 \\
\hline
\end{tabular}

Sources: Ministry of Industrialization and Handicrafts; and Ministry of Energy and Mining. 
Table 11. Madagascar: Economic Activities of Manufacturing Enterprises in the Export Processing Zones (EPZs), 1994-96

\begin{tabular}{|c|c|c|c|}
\hline & 1994 & 1995 & 1996 \\
\hline Number of manufacturing enterprises operating in EPZs & 7 & 98 & 125 \\
\hline (in percent) $1 /$ & 1.1 & 1.8 & 1.8 \\
\hline \multicolumn{4}{|l|}{ Of which } \\
\hline Textiles and hides & 43 & 64 & 69 \\
\hline Wood processing & 8 & 8 & 10 \\
\hline Food, beverages, and tobacco & 3 & 5 & 3 \\
\hline Other (mainly jewelry and handicrafts) & 8 & 14 & 43 \\
\hline Total number of employees & 17,400 & 29,615 & 36,700 \\
\hline (in percent) $1 /$ & 12.0 & 20.0 & 23.0 \\
\hline Wages paid (in billions of Malagasy francs) & 25 & 66 & 104 \\
\hline (in percent) $1 /$ & 9.1 & 15.3 & 20.0 \\
\hline Value added (in billions of Malagasy francs) & 58 & 127 & 156 \\
\hline (in percent) $1 /$ & 5.5 & 9.3 & 9.5 \\
\hline Exports (in billions of Malagasy francs) & 164 & 392 & 590 \\
\hline (in percent) $1 /$ & 24.0 & 38.5 & 22.7 \\
\hline Imports (in billions of Malagasy francs) & 82 & 191 & 293 \\
\hline (in percent) $1 /$ & 16.0 & 13.1 & 23.7 \\
\hline
\end{tabular}

Source: Project Madio: "Le Secteur Industriel Formel à Madagascar: Caractéristiques, Performances Perspectives." various issues, 1995-97.

$1 /$ In percent of the total for manufacturing sector. 
Table 12. Madagascar: Number of Enterprises with Operating Permits Under the Export Processing Zone Regime, 1994-98

\begin{tabular}{|c|c|c|c|c|c|c|c|c|}
\hline & \multirow[t]{3}{*}{1994} & \multirow[t]{3}{*}{1995} & \multirow[t]{3}{*}{1996} & \multirow[t]{3}{*}{1997} & \multirow[t]{3}{*}{1998} & \multicolumn{3}{|c|}{$1994-98$} \\
\hline & & & & & & \multicolumn{3}{|c|}{ Projections } \\
\hline & & & & & & Firms & Employees & Investments $1 /$ \\
\hline \multicolumn{9}{|l|}{ Sectors } \\
\hline Agro-food processing & 10 & 1 & 3 & $\ldots$ & $\ldots$ & 26 & 2,024 & 53.4 \\
\hline Textiles & 9 & 19 & 11 & $\ldots$ & $\ldots$ & 99 & 25,281 & 134.1 \\
\hline Hides and skins & 1 & 0 & 0 & $\ldots$ & $\ldots$ & 5 & 707 & 113 \\
\hline Wood processing & 2 & 4 & 2 & $\ldots$ & $\ldots$ & 16 & 1,312 & 38.5 \\
\hline Data processing & 6 & 3 & 7 & $\ldots$ & $\ldots$ & 21 & 1,397 & 9.3 \\
\hline Chemicals & 1 & 1 & 2 & $\ldots$ & $\ldots$ & 6 & 297 & 15.7 \\
\hline Electrical and Mechanical & 0 & 0 & 1 & $\ldots$ & $\ldots$ & 4 & 129 & 5 \\
\hline Mineral Processing & 0 & 4 & 0 & $\ldots$ & $\ldots$ & 6 & 424 & 32.4 \\
\hline Handicrafts & 2 & 6 & 2 & $\ldots$ & $\ldots$ & 15 & 2,741 & 13 \\
\hline Jewelry & 1 & 1 & 1 & $\ldots$ & $\ldots$ & 10 & 458 & 9.3 \\
\hline $\begin{array}{l}\text { Enterprises promoting } \\
\text { and managing EPZs }\end{array}$ & 0 & 0 & 0 & $\ldots$ & $\ldots$ & 1 & 66 & 111.2 \\
\hline Total & 32 & 39 & 29 & & & 209 & 34,836 & 433.7 \\
\hline
\end{tabular}

Source: Malagasy authorities.

1/ In billions of Malagasy francs. 
Table 13. Madagascar: Production and Export of Major Minerals, 1994-98

\begin{tabular}{|c|c|c|c|c|c|}
\hline & 1994 & 1995 & 1996 & 1997 & $\begin{array}{r}1998 \\
\text { Est. }\end{array}$ \\
\hline \multicolumn{6}{|c|}{ Production (thousand tons) } \\
\hline Chromite & 75.0 & 128.5 & 110.1 & 117.8 & 119.9 \\
\hline Graphite & 14.7 & 16.5 & 14.3 & 14.0 & 14.3 \\
\hline \multicolumn{6}{|c|}{ Exports (thousand tons) } \\
\hline Chromite & 90.0 & 128.5 & 110.1 & 117.8 & 119.9 \\
\hline Graphite & 15.6 & 16.5 & 14.3 & 14.0 & 14.3 \\
\hline \multicolumn{6}{|c|}{ Export value (millions of SDRs) } \\
\hline Chromite & 3.8 & 7.7 & 6.8 & 6.5 & 6.3 \\
\hline Graphite & 5.5 & 6.3 & 5.4 & 5.1 & 5.4 \\
\hline \multicolumn{6}{|c|}{ Unit value (SDRs per ton) } \\
\hline Chromite & 42.8 & 59.9 & 61.4 & 55.4 & 52.5 \\
\hline Graphite & 354.9 & 380.8 & 376.7 & 360.7 & 377.6 \\
\hline
\end{tabular}

Source: Ministry of Energy and Mining. 
Table 14. Madagascar: Tourism Indicators, 1994-98

\begin{tabular}{|c|c|c|c|c|c|}
\hline & 1994 & 1995 & 1996 & 1997 & $\begin{array}{r}1998 \\
\text { Est. }\end{array}$ \\
\hline Number of tourists & 65,839 & 74,619 & 82,681 & 104,931 & 133,500 \\
\hline Annual growth (in percent) & 18.9 & 13.3 & 10.8 & 26.9 & 27.2 \\
\hline Hotel capacity (number of rooms) & 4,000 & 5,000 & 6,066 & 7,060 & 8,090 \\
\hline Average capacity utilization & 525 & 550 & 580 & 550 & 550 \\
\hline Number of tourist nights & 722,718 & 770,570 & 812,314 & $1,464,210$ & $1,742,175$ \\
\hline Annual growth (in percent) & 18.7 & 6.6 & 5.4 & 80.3 & 19.0 \\
\hline Average length of stay (days) & 11.0 & 10.3 & 9.8 & 18.0 & 18.0 \\
\hline $\begin{array}{l}\text { Foreign exchange receipts from } \\
\text { tourism (in millions of SDRs) }\end{array}$ & 32.2 & 38.0 & 44.6 & 52.9 & 68.0 \\
\hline Annual growth (in percent) & 13.4 & 18.0 & 17.4 & 18.6 & 28.5 \\
\hline
\end{tabular}

Source: Ministry of Tourism.

1/ Coverage is not complete. 
Table 15. Madagascar: Production and Consumption of Electricity, 1994-98

(In millions of kilowatt-hours)

\begin{tabular}{|c|c|c|c|c|c|}
\hline & 1994 & 1995 & 1996 & 1997 & $\begin{array}{r}1998 \\
\text { Est. }\end{array}$ \\
\hline Production 1/ & 546 & 552 & 563 & 616 & 642 \\
\hline Hydroelectric & 389 & 415 & 442 & 489 & 503 \\
\hline Thermal & 157 & 137 & 121 & 128 & 139 \\
\hline Consumption & 450 & 439 & 441 & 485 & 528 \\
\hline Public lighting & 7 & 7 & 7 & 8 & 8 \\
\hline Households & 162 & 169 & 183 & 200 & 226 \\
\hline Other & 281 & 264 & 252 & 278 & 293 \\
\hline
\end{tabular}

Source: Ministry of Energy and Mining.

1/ Electric power generated by the electricity and water company, that is, excluding electricity generated by industries for their own consumption. 
Table 16. Madagascar: Prices of Petroleum Products, 1994-98 1/

(In Malagasy francs per liter)

\begin{tabular}{|c|c|c|c|c|c|c|c|c|c|c|c|c|c|c|c|c|c|}
\hline & \multirow{2}{*}{$\begin{array}{c}1994 \\
\text { Dec. }\end{array}$} & \multicolumn{4}{|c|}{1995} & \multicolumn{4}{|c|}{1996} & \multicolumn{4}{|c|}{1997} & \multicolumn{4}{|c|}{1998} \\
\hline & & Mar. & Jun. & Sep. & $\overline{\text { Dec. }}$. & Mar. & Jun. & Sep. & $\overline{\text { Dec. }}$ & Mar. & Jun. & Sep. & $\overline{\text { Dec. }}$ & Mar. & Jun. & Sep. & $\overline{\text { Dec. }}$ \\
\hline Gasoline (premium) & 2,110 & 1,631 & 1,940 & 1,931 & 1,930 & 1,860 & 2,120 & 2,150 & 2,180 & 2,236 & 2,383 & 2,383 & 2,383 & 2,530 & 2,530 & 2,530 & 2,530 \\
\hline Gasoline (regular) & 1,240 & 1,503 & 1,630 & 1,640 & 1,610 & 1,550 & 1,800 & 1,830 & 1,860 & 1,913 & 2,000 & 2,000 & 2,000 & 2,106 & 2,090 & 2,090 & 2,090 \\
\hline Gas oil & 727 & 1,226 & 1,280 & 1,340 & 1,320 & 1,340 & 1,510 & 1,460 & 1,500 & 1,553 & 1,650 & 1,650 & 1,650 & 1,727 & 1,700 & 1,700 & 1,700 \\
\hline Kerosene & 513 & 1,090 & 1,140 & 1,210 & 1,190 & 1,220 & 1,380 & 1,300 & 1,340 & $\ldots$ & 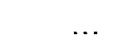 & $\ldots$ & $\ldots$ & $\ldots$ & $\ldots$ & $\ldots$ & $\ldots$ \\
\hline Natural gas 2/ & 25,000 & 34,000 & 34,000 & 25,000 & 25,000 & 25,000 & 29,500 & 29,500 & 34,000 & 36,000 & 34,000 & 34,000 & 34,000 & 42,000 & $\ldots$ & $\ldots$ & $\ldots$ \\
\hline
\end{tabular}

Source: Ministry of Energy and Mining.

1/ Wholesale prices from storage facilities; retail prices are higher by a small, fixed-profit margin. Prices recorded in the capital city only. 2/ Prices are for a 12.5 kilograms container. 
Table 17. Madagascar: Population and Labor Force, 1990-98

(In thousands, unless otherwise indicated)

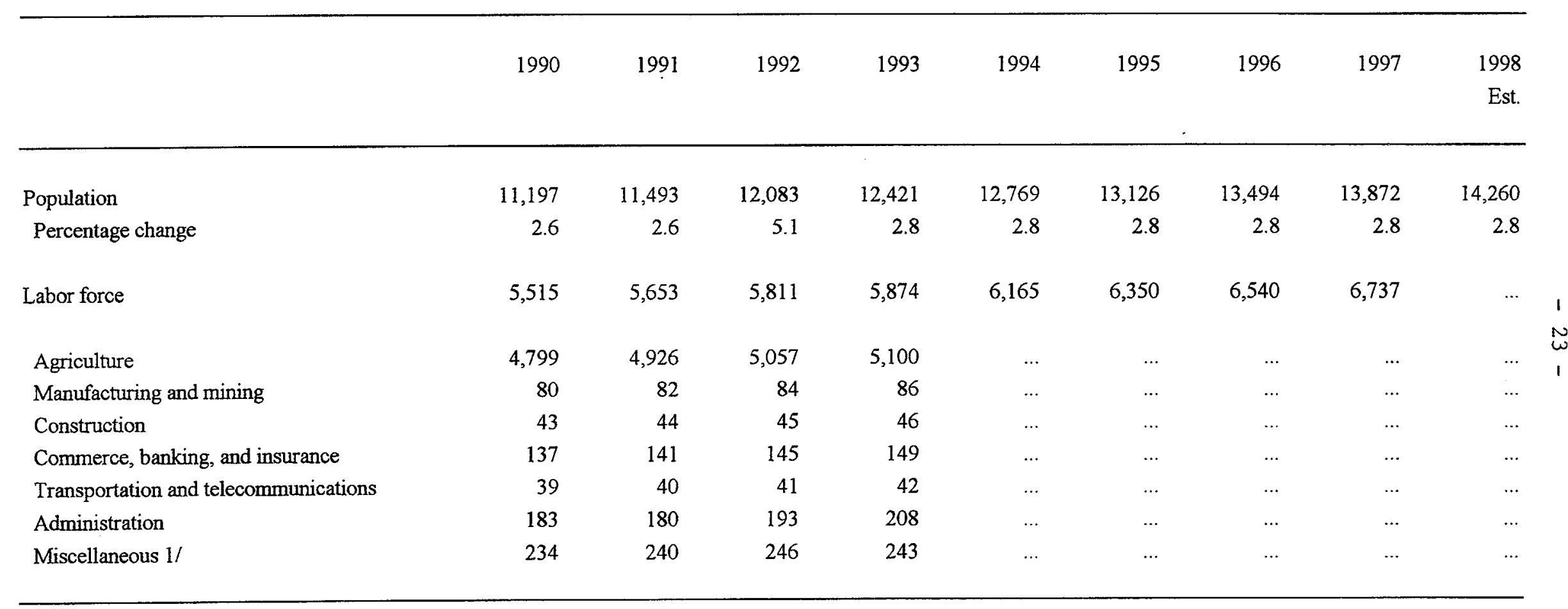

Sources: National Institute of Statistics; and National Social Security Fund.

1/ No data have been published by the National Social Security Fund after 1993.

2/ Includes craftspeople and domestics. 
Table 18. Madagascar: Distribution of Population and Civil Servants, 1990-98

$$
\text { (In percent of total) }
$$

\begin{tabular}{lrrrr}
\hline & $\begin{array}{c}\text { Population } \\
\text { Region }\end{array}$ & \multicolumn{3}{c}{ Civil Servants } \\
\cline { 4 - 5 } & Est. & 1997 & 1997 & 1998 \\
& & & & \\
\hline Antananarivo & 29.0 & 42.2 & 43.0 & 42.8 \\
Antsiranana & 7.8 & 6.8 & 6.3 & 6.3 \\
Fianarantsoa & 21.7 & 16.8 & 17.1 & 16.8 \\
Mahajanga & 11.1 & 9.9 & 9.6 & 9.7 \\
Toamasina & 15.8 & 12.8 & 12.8 & 12.8 \\
Toliara & 14.6 & 11.4 & 11.2 & 11.6 \\
& & & & \\
Total & 100.0 & 100.0 & 100.0 & 100.0 \\
& & & & \\
\hline
\end{tabular}

Sources: Ministry of Finance and Economy; and Ministry of Budget and Development of Autonomous Provinces. 
Table 19. Madagascar: Minimum and Maximum Monthly Wages, 1984-98 1/

(In Malagasy francs)

\begin{tabular}{|c|c|c|c|c|}
\hline & \multicolumn{2}{|c|}{ Agricultural 2/ } & \multicolumn{2}{|c|}{ Nonagricultural 3/ } \\
\hline & Minimum & Maximum & Minimum & Maximum \\
\hline January 1984-July 1984 & 18,000 & 72,000 & 17,675 & 70,718 \\
\hline August 1984-June 1985 & 18,900 & 74,100 & 18,563 & 72,701 \\
\hline July 1985-September 1986 & 20,460 & 77,700 & 20,331 & 76,317 \\
\hline October 1986-February 1987 & 22,200 & 80,400 & 21,804 & 78,969 \\
\hline March 1987-June 1987 & 24,300 & 86,100 & 23,867 & 84,568 \\
\hline July 1987-January 1988 & 26,080 & 92,400 & 25,618 & 90,772 \\
\hline February 1988-February 1989 & 29,300 & 100,780 & 28,772 & 99,006 \\
\hline March 1989-February 1990 & 32,840 & 111,740 & 32,274 & 109,770 \\
\hline March 1990-January 1991 & 35,600 & 121,100 & 35,013 & 119,095 \\
\hline February 1991-December 1993 & 41,200 & 140,180 & 40,490 & 137,728 \\
\hline January 1994-February 1995 & 64,236 & 158,772 & 63,313 & 155,997 \\
\hline March 1995-October 1996. & 112,435 & 244,080 & 110,550 & 239,993 \\
\hline November 1996-March 1998 & 123,778 & 268,704 & 121,591 & 263,947 \\
\hline Since April 1998 & 142,280 & 308,880 & 134,860 & 303,639 \\
\hline
\end{tabular}

Source: Ministry of Civil Service, Labor, and Social Laws.

1/ The "minimum" indicates the minimum wage in the lowest wage category, and the "maximum" indicates the minimum wage in the highest wage category.

2/ On the basis of 200 working hours per month.

3/ On the basis of 173.33 working hours per month. 
Table 20. Madagascar: Consumer Price Index, 1994-98

(Period averages; August 1971-July $1972=100$ )

\begin{tabular}{|c|c|c|c|c|c|c|c|c|c|}
\hline & \multirow[t]{2}{*}{ Weights } & \multirow[t]{2}{*}{1994} & \multirow[t]{2}{*}{1995} & \multirow[t]{2}{*}{1996} & \multirow[t]{2}{*}{1997} & \multicolumn{4}{|c|}{1998} \\
\hline & & & & & & I & II & III & IV $1 /$ \\
\hline & \multicolumn{9}{|c|}{ (Traditional households) 2} \\
\hline Food & 60.35 & 2,208 & 3,326 & 3,959 & 4,121 & 4,382 & 4,312 & 4,285 & 4,457 \\
\hline Lighting and fuel & 9.14 & 2,022 & 2,656 & 3,344 & 3,662 & 4,042 & 4,051 & 3,950 & 4,004 \\
\hline Domestic services & 1.82 & 638 & 949 & 1,052 & 1,052 & 1,052 & 1,052 & 1,052 & 1,052 \\
\hline Maintenance, clothing and medicine & 14.85 & 1,930 & 2,912 & 3,334 & 3,490 & 3,674 & 3,717 & 3,737 & 3,753 \\
\hline Miscellaneous & 13.84 & 2,296 & 3,466 & 4,327 & 4,476 & 4,408 & 4,707 & 4,838 & 4,846 \\
\hline General index & 100.00 & 2,133 & 3,179 & 3,808 & 3,979 & 4,189 & 4,195 & 4,191 & 4,301 \\
\hline \multirow[t]{2}{*}{ Annual change (in percent) } & $\ldots$ & 39.1 & 49.0 & 19.8 & 4.5 & 5.0 & 7.1 & 6.8 & 5.4 \\
\hline & \multicolumn{9}{|c|}{ (Modern households) } \\
\hline Food & 45.16 & 2,156 & 3,106 & 3,875 & 4,271 & 4,737 & 4,968 & 5,103 & 5,156 \\
\hline Lighting and fuel & 6.08 & 1,393 & 2,136 & 2,328 & 2,589 & 3,133 & 3,157 & 3,246 & 3,308 \\
\hline Domestic services & 11.26 & 878 & 1,248 & 1,371 & 1,371 & 1,371 & 1,371 & 1,371 & 1,371 \\
\hline Maintenance, clothing and medicine & 17.99 & 1,969 & 2,890 & 3,369 & 3,639 & 3,909 & 3,955 & 3,981 & 4,011 \\
\hline Miscellaneous & 19.51 & 1,847 & 2,822 & 3,385 & 3,586 & 3,863 & 3,767 & 3,776 & 3,841 \\
\hline General index & 100.00 & 1,872 & 2,744 & 3,312 & 3,595 & 3,941 & 4,036 & 4,109 & 4,155 \\
\hline \multirow[t]{2}{*}{ Annual change (in percent) } & $\ldots$ & 30.5 & 46.6 & 20.7 & 8.5 & 12.5 & 15.3 & 13.7 & 10.5 \\
\hline & \multicolumn{9}{|c|}{ (Household combined) 3/ } \\
\hline Combined consumer price index & 100.00 & 2,068 & 3,070 & 3,684 & 3,883 & 4,127 & 4,155 & 4,170 & 4,265 \\
\hline
\end{tabular}

Source: National Institute of Statistics.

1/ Through end-November 1998.

$2 /$ Low-income househoids.

3/ Weights of 0.75 and 0.25 are applied to the index of traditional households and to the index of modern households, respectively. 
Table 21. Madagascar: Central Government Financial Operations, 1994-98

(In billions of Malagasy francs)

\begin{tabular}{|c|c|c|c|c|c|}
\hline & 1994 & 1995 & 1996 & 1997 & $\begin{array}{r}1998 \\
\text { Est. }\end{array}$ \\
\hline Total revenue and grants & 1,036 & 1,542 & 2,091 & 2,703 & 2,875 \\
\hline Total revenue & 762 & 1,150 & 1,407 & 1,747 & 2,183 \\
\hline Budgetary revenue & 748 & 1,149 & 1,405 & 1,747 & 2,166 \\
\hline Of which: tax revenue & 702 & 1,121 & 1,374 & 1,688 & 2,068 \\
\hline Extrabudgetary and capital revenue & 14 & 1 & 3 & 0 & 17 \\
\hline Grants & 274 & 392 & 683 & 956 & 692 \\
\hline Current grants & 39 & 31 & 116 & 378 & 52 \\
\hline Project grants & 235 & 362 & 567 & 579 & 640 \\
\hline Total expenditure & 1,804 & 2,374 & 2,883 & 3,137 & 3,702 \\
\hline Current expenditure & 1,168 & 1,523 & 1,704 & 1,960 & 2,089 \\
\hline Budgetary expenditure & 1,067 & 1,498 & 1,679 & 1,802 & 1,990 \\
\hline Personnel & 330 & 444 & 523 & 670 & 836 \\
\hline Other noninterest expenditure & 245 & 366 & 397 & 583 & 686 \\
\hline Foreign interest obligations & 462 & 624 & 629 & 504 & 384 \\
\hline Domestic interest obligations & 30 & 64 & 131 & 45 & 84 \\
\hline Extrabudgetary expenditure & 101 & 25 & 25 & 120 & 99 \\
\hline Exceptional expenditure 1/ & 0 & 0 & 0 & 39 & 0 \\
\hline Capital expenditure & 636 & 850 & 1,180 & 1,177 & 1,612 \\
\hline Domestic financing & 193 & 197 & 284 & 275 & 512 \\
\hline Foreign financing & 443 & 654 & 895 & 902 & 1,100 \\
\hline \multicolumn{6}{|l|}{ Overall balance (commitment basis) } \\
\hline Including grants & -768 & -832 & -793 & -434 & -827 \\
\hline Excluding grants & $-1,042$ & $-1,224$ & $-1,476$ & $-1,390$ & $-1,519$ \\
\hline Changes in domestic arrears & -12 & 45 & 3 & -139 & -101 \\
\hline Overall balance (cash basis) & -779 & -787 & -790 & -573 & -928 \\
\hline Net cost of structural reforms & 0 & 0 & 0 & 0 & -162 \\
\hline Total overall balance (cash basis) & -779 & -787 & -790 & -573 & $-1,090$ \\
\hline Financing & 779 & 787 & 790 & 573 & 1,090 \\
\hline Foreign (net) & 566 & 825 & 805 & 692 & 207 \\
\hline Drawings & 217 & 299 & 329 & 1,029 & 549 \\
\hline Amortization due & -595 & -795 & -604 & -509 & -669 \\
\hline Change in external arrears & 944 & 1,320 & 1,080 & $-5,460$ & $-2,556$ \\
\hline External debt relief & 0 & 0 & 0 & 5,632 & 2,883 \\
\hline Domestic (net) & 213 & -38 & -15 & -119 & 879 \\
\hline Banking system & 201 & -43 & -45 & -146 & 726 \\
\hline Nonbanking system & 12 & 5 & 30 & 27 & 153 \\
\hline Divestment revenuc & 0 & 0 & 0 & 0 & 4 \\
\hline
\end{tabular}

Sources: Ministry of Finance and Economy; Ministry of Budget and Development of Autonomous Provinces; and Fund staff estimates.

1/ Once-and-for-all outlays, mainly related to structural reforms. Beginning with 1998, expenditures related to structural reforms are no longer recorded as current outlays. 
Table 22. Madagascar: Fiscal Indicators, 1994-98

(In percent of GDP)

\begin{tabular}{|c|c|c|c|c|c|}
\hline & 1994 & 1995 & 1996 & 1997 & $\begin{array}{r}1998 \\
\text { Est. }\end{array}$ \\
\hline Total revenue and grants & 11.3 & 11.4 & 12.9 & 15.0 & 14.1 \\
\hline Total revenue & 8.3 & 8.5 & 8.7 & 9.7 & 10.7 \\
\hline Of which: tax revenue & 7.7 & 8.3 & 8.5 & 9.4 & 10.1 \\
\hline Grants & 3.0 & 2.9 & 4.2 & 5.3 & 3.4 \\
\hline Current grant & 0.4 & 0.2 & 0.7 & 2.1 & 0.3 \\
\hline Project grants & 2.6 & 2.7 & 3.5 & 3.2 & 3.1 \\
\hline Current expenditure $1 /$ & 12.8 & 11.3 & 10.5 & 10.9 & 10.2 \\
\hline Noninterest expenditure & 7.4 & 6.2 & 5.8 & 7.8 & 8.0 \\
\hline Personnel & 3.6 & 3.3 & 3.2 & 3.7 & 4.1 \\
\hline Other & 3.8 & 2.9 & 2.6 & 4.1 & 3.9 \\
\hline Interest obligations & 5.4 & 5.1 & 4.7 & 3.0 & 2.3 \\
\hline Capital expenditure & 7.0 & 6.3 & 7.3 & 6.5 & 7.9 \\
\hline Primary balance & -3.0 & -1.1 & -0.2 & 0.6 & -1.8 \\
\hline \multicolumn{6}{|l|}{ Overall balance (commitment basis) } \\
\hline Including grants & -8.4 & -6.2 & -4.9 & -2.4 & -4.1 \\
\hline Excluding grants & -11.4 & -9.1 & -9.1 & -7.7 & -7.4 \\
\hline Change in domestic arrears & -0.1 & 0.3 & 0.0 & -0.8 & -0.5 \\
\hline Overall balance (cash basis) & -8.5 & -5.8 & -4.9 & -3.2 & -4.5 \\
\hline Net cost of structural reforms & 0.0 & 0.0 & 0.0 & 0.0 & -0.8 \\
\hline Total overall balance (cash basis) & -8.5 & -5.8 & -4.9 & -3.2 & -5.3 \\
\hline Financing & 8.5 & 5.8 & 4.9 & 3.2 & 5.3 \\
\hline Foreign (net) & 6.2 & 6.1 & 5.0 & 3.8 & 1.0 \\
\hline Drawings minus amortization & -4.1 & -3.7 & -1.7 & 2.9 & -0.6 \\
\hline Change in external arrears & 10.3 & 9.8 & 6.7 & -30.2 & -12.5 \\
\hline External debt relief & 0.0 & 0.0 & 0.0 & 31.2 & 14.1 \\
\hline Financing gap & 0.0 & 0.0 & 0.0 & 0.0 & 0.0 \\
\hline Domestic (net) & 2.3 & -0.3 & -0.1 & -0.7 & 4.3 \\
\hline Divestment revenue & 0.0 & 0.0 & 0.0 & 0.0 & 0.0 \\
\hline \multicolumn{6}{|l|}{ Memorandum items: } \\
\hline Education expenditure & 1.9 & 1.9 & 1.6 & 1.6 & 2.0 \\
\hline Health expenditure & 0.9 & 1.0 & 0.8 & 1.0 & 1.4 \\
\hline Nominal GDP (in billions of Malagasy francs) & $9,131.2$ & $13,478.7$ & $16,224.4$ & $18,050.8$ & $20,397.8$ \\
\hline
\end{tabular}

Sources: Ministry of Finance and Economy; Ministry of Budget and Development of Autonomous Provinces; Fund staff estimates; and World Bank estimates.

1/ Including budgetary, extrabudgetary, and exceptional expenditures. 
Tableau 23. Madagascar: Budgetary Revenue, 1994-98

(In billions of Malagasy francs)

\begin{tabular}{|c|c|c|c|c|c|}
\hline & 1994 & 1995 & 1996. & 1997 & $\begin{array}{r}1998 \\
\text { Est. }\end{array}$ \\
\hline Tax revenue & 702.2 & $1,120.9$ & $1,374.4$ & $1,687.9$ & $2,068.1$ \\
\hline Taxes on net income & 150.7 & 167.8 & 258.4 & 329.3 & 355.7 \\
\hline Companies & 68.5 & 83.2 & 163.6 & 201.5 & 204.3 \\
\hline Individuals & 40.6 & 58.6 & 59.0 & 87.1 & 99.4 \\
\hline Of which: tax on wages and salaries (IRSA) & 31.9 & 45.9 & 45.7 & 67.1 & 75.9 \\
\hline Other & 41.6 & 26.0 & 35.8 & 40.7 & 52.0 \\
\hline Taxes on property & 11.2 & 14.0 & 17.4 & 26.9 & 19.5 \\
\hline Of which: registration dues & 9.4 & 11.9 & 15.0 & 23.5 & 16.2 \\
\hline Taxes on goods and services & 201.5 & 298.7 & 341.6 & 369.6 & 501.0 \\
\hline Value added tax (VAT) & 177.5 & 242.3 & 166.8 & 177.6 & 291.0 \\
\hline Excises & 6.0 & 0.8 & 74.4 & 65.5 & 80.2 \\
\hline Fiscal monopoly profits & 11.2 & 46.4 & 91.0 & 112.3 & 110.8 \\
\hline Other & 6.8 & 9.2 & 9.4 & 14.2 & 19.0 \\
\hline Taxes on foreign trade & 335.5 & 633.7 & 750.3 & 947.6 & $1,179.4$ \\
\hline Import duties & 320.0 & 589.8 & 731.6 & 940.9 & $1,179.4$ \\
\hline Customs duty & 51.8 & 81.3 & 102.9 & 131.5 & 155.4 \\
\hline Petroleum products (TUPP) & 19.1 & 78.9 & 155.7 & 187.4 & 142.0 \\
\hline Other & 249.1 & 429.6 & 473.0 & 622.0 & 882.0 \\
\hline Export duties & 15.5 & 43.9 & 18.7 & 6.7 & 0.0 \\
\hline Export duty & 9.8 & 43.8 & 18.7 & 6.7 & 0.0 \\
\hline Export surcharge & 4.5 & 0.1 & 0.0 & 0.0 & 0.0 \\
\hline Tax on coffee and cloves & -- & 0.0 & 0.0 & 0.0 & 0.0 \\
\hline Special levy on FNUP receipts $1 /$ & 1.2 & 0.0 & 0.0 & 0.0 & 0.0 \\
\hline Other taxes & 3.3 & 6.7 & 6.7 & 14.5 & 12.5 \\
\hline Nontax revenue & 46.2 & 27.9 & 30.4 & 58.9 & 97.5 \\
\hline Property income & 18.1 & 10.0 & 8.5 & 10.6 & 3.0 \\
\hline Other & 25.6 & 15.4 & 21.9 & 48.3 & 94.5 \\
\hline Fishing licenses & 2.5 & 2.5 & 0.0 & 0.0 & 0.0 \\
\hline Total budgetary revenue & 748.4 & $1,148.7$ & $1,404.8$ & $1,746.8$ & $2,165.6$ \\
\hline
\end{tabular}

Sources: Ministry of Finance and Economy; Ministry of Budget and Development of Autonomous Provinces; and Fund staff estimates.

1/ FNUP = export stabilization fund. 
Table 24. Madagascar: Current Budgetary Expenditure, 1994-98

\begin{tabular}{|c|c|c|c|c|c|}
\hline & 1994 & 1995 & 1996 & 1997 & $\begin{array}{r}1998 \\
\text { Est. }\end{array}$ \\
\hline Economic classification & \multicolumn{5}{|c|}{ (In billions of Malagasy francs) } \\
\hline Current expenditure $1 /$ & $1,168.1$ & $1,523.4$ & $1,704.0$ & $1,960.0$ & $2,089.4$ \\
\hline Personnel 2/ & 329.5 & 444.5 & 523.0 & 669.6 & 836.0 \\
\hline Of which: wages and salaries & 307.0 & 428.8 & 509.4 & 637.4 & 764.3 \\
\hline Goods and services & 131.2 & 212.0 & 184.2 & 336.7 & 339.0 \\
\hline Interest on public debt & 491.9 & 687.9 & 759.2 & 548.9 & 467.6 \\
\hline Of which: foreign & 462.2 & 624.3 & 628.6 & 503.5 & 384.0 \\
\hline Current transfers and subsidies $3 /$ & 114.2 & 153.8 & 212.7 & 246.3 & 347.4 \\
\hline \multicolumn{6}{|l|}{ Functional classification } \\
\hline Budgetary expenditure & $1,066.8$ & $1,498.0$ & $1,679.1$ & $1,801.6$ & $1,990.0$ \\
\hline General public services 4/ & 181.4 & 261.9 & 416.9 & 688.7 & 741.2 \\
\hline Of which: defense & 84.6 & 115.9 & 200.8 & 267.4 & 287.2 \\
\hline Education & 146.5 & 188.2 & 187.7 & 258.5 & 351.5 \\
\hline Of which: universities & 50.1 & 40.1 & 43.1 & 67.2 & 64.4 \\
\hline Health & 53.1 & 73.5 & 65.9 & 98.7 & 124.9 \\
\hline Social and community services & 9.3 & 10.9 & 14.6 & 19.3 & 21.9 \\
\hline \multicolumn{5}{|l|}{ Of which } & 103.1 \\
\hline Agriculture 5/ & 29.2 & 12.5 & 49.2 & 68.7 & 49.4 \\
\hline Public works & 6.4 & 10.9 & 8.2 & 16.6 & 11.7 \\
\hline Unclassified $6 /$ & 577.1 & 811.0 & 915.3 & 614.3 & 647.4 \\
\hline Of which: interest on government debt & 491.9 & 687.9 & 759.2 & 548.9 & 467.6 \\
\hline Memorandum items: & \multicolumn{5}{|c|}{ (In percent of GDP) } \\
\hline Noninterest budgetary expenditure & 6.3 & 6.0 & 5.7 & 6.9 & 7.5 \\
\hline Social sector current expenditure $7 /$ & 2.3 & 2.0 & 1.7 & 2.1 & 2.4 \\
\hline Of which : health & 0.6 & 0.5 & 0.4 & 0.5 & 0.6 \\
\hline Defense & 0.9 & 0.9 & 1.2 & 1.5 & 1.4 \\
\hline
\end{tabular}

Sources: Ministry of Finance and Economy; Ministry of Budget and Development of Autonomous Provinces; and Fund staff estimates.

1/ Differs from budgetary expenditure total because of inclusion of extrabudgetary and additional expenditures.

2/ Including travel expenses but excluding medical expenses.

3/ Including medical expenses of employees.

4/ Including national defense and security expenditures.

$5 /$ Including livestock, fishing,water, and forestry.

6/ Including revenue sharing with local government and payments to international organizations.

$7 /$ Including expenditure on education, health, and social and community services. 
Table 25. Madagascar: Central Government Personnel Expenditure and Number of Civil Servants, 1994-98

(In thousands of Malagasy francs)

\begin{tabular}{|c|c|c|c|c|c|}
\hline & 1994 & 1995 & 1996 & 1997 & $\begin{array}{r}1998 \\
\text { Est. }\end{array}$ \\
\hline Personnel expenditure (commitment basis) $1 / 2 /$ & 329.5 & 444.5 & 523.0 & 669.6 & 836.0 \\
\hline Annual change in percent & 23.6 & 34.9 & 17.7 & 28.0 & 24.9 \\
\hline In percent of current budgetary expenditure & 30.9 & 29.7 & 31.1 & 37.2 & 42.0 \\
\hline In percent of total budgetary expenditure $3 /$ & 23.9 & 23.1 & 24.0 & 29.2 & 29.8 \\
\hline In percent of total government expenditure & 18.3 & 18.7 & 18.2 & 21.3 & 22.6 \\
\hline In percent of GDP & 3.6 & 3.3 & 3.2 & 3.7 & 4.1 \\
\hline \multicolumn{6}{|l|}{ Civil servants (number at end-December) $4 /$} \\
\hline Civilian & 94,683 & 92,714 & 93,476 & 93,971 & ... \\
\hline Military & 22,076 & 21,475 & 21,475 & 21,760 & $\cdots$ \\
\hline Total & 116,759 & 114,189 & 114,951 & 115,731 & $\cdots$ \\
\hline Annual change in percent & -0.9 & -2.2 & 0.7 & 0.7 & $\ldots$ \\
\hline \multicolumn{6}{|l|}{ Memorandum items: } \\
\hline \multicolumn{6}{|l|}{ Annual average civil servants' salary } \\
\hline In thousand of Malagasy francs & $2,629.3$ & $3,755.2$ & $4,431.5$ & $5,507.6$ & $\ldots$ \\
\hline Index, $1990=1005 /$ & 123.3 & 118.1 & 116.4 & 138.4 & .. \\
\hline Annual change in percent & -7.0 & -4.2 & -1.5 & 18.9 & ... \\
\hline
\end{tabular}

Sources: Ministry of Finance and Economy; Ministry of Budget and Development of Autonomous Provinces; and Fund staff estimates.

1/ Including only regular staff. Expenditure on some categories of temporary personel is included in goods and services outlays.

2/ Excluding medical expenses.

3/ Consisting of current budgetary expenditure and capital budgetary expenditure.

4/ Data on actual positions filled, which may differ from other sources.

5/ Annual average of civil servants' salary deflated by the consumer price index (traditional). 
Table 26 . Madagascar: Structure and Adjustment of Public Sector Salaries, 1994-98

(In thousands of Malagasy francs)

\begin{tabular}{|c|c|c|c|c|c|c|c|}
\hline & $\begin{array}{l}\text { Jan. } 1994 \text { - } \\
\text { Dec. } 1994\end{array}$ & $\begin{array}{l}\text { Jan. } 1995 \text { - } \\
\text { Mar. } 1995\end{array}$ & $\begin{array}{l}\text { Apr. } 1995 \text { - } \\
\text { Dec. } 1995\end{array}$ & $\begin{array}{l}\text { Jan. } 1996 \text { - } \\
\text { Dec. } 1996\end{array}$ & $\begin{array}{l}\text { Jan. } 1997 \text { - } \\
\text { Dec. } 1997\end{array}$ & $\begin{array}{l}\text { Jan.1998 - } \\
\text { Apr. } 1998\end{array}$ & $\begin{array}{r}\text { Since } \\
\text { May } 1998\end{array}$ \\
\hline \multicolumn{8}{|l|}{ Category I } \\
\hline Minimum & 77.1 & 110.6 & 129.0 & 135.3 & 208.9 & 239.3 & $-\quad \ldots$ \\
\hline Maximum & 126.6 & 162.1 & 192.9 & 203.4 & 287.0 & 317.5 & $\ldots$ \\
\hline (Midpoint) & 101.9 & 136.4 & 161.0 & 169.4 & 248.0 & 278.4 & $\ldots$ \\
\hline \multicolumn{8}{|l|}{ Category II } \\
\hline Minimum & 97.3 & 131.8 & 157.3 & 165.3 & 242.8 & 273.5 & $\ldots$ \\
\hline Maximum & 156.6 & 195.0 & 228.1 & 241.1 & 331.1 & 365.7 & $\ldots$ \\
\hline (Midpoint) & 127.0 & 163.4 & 192.7 & 203.2 & 287.0 & 319.6 & $\ldots$ \\
\hline \multicolumn{8}{|l|}{ Category III } \\
\hline Minimum & 110.7 & 145.9 & 174.5 & 183.7 & 264.2 & 295.2 & 328.6 \\
\hline Maximum & 221.0 & 261.9 & 295.5 & 314.0 & 417.7 & 460.5 & 484.6 \\
\hline (Midpoint) & 165.9 & 203.9 & 235.0 & 248.9 & 341.0 & 377.9 & 406.6 \\
\hline \multicolumn{8}{|c|}{ Categories $I V$ and $V$} \\
\hline Minimum & $140.6^{\circ}$ & 177.9 & 210.3 & 221.9 & 308.4 & 341.4 & 357.6 \\
\hline Maximum & 329.0 & 371.4 & 405.1 & 432.8 & 559.5 & 613.0 & 660.6 \\
\hline (Midpoint) & 234.8 & 274.7 & 307.7 & 327.4 & 434.0 & 477.2 & 509.1 \\
\hline \multicolumn{8}{|c|}{ Categories VI and VII } \\
\hline Minimum & 170.7 & 209.6 & 242.9 & 257.1 & 350.0 & 385.6 & 414.9 \\
\hline Maximum & 374.6 & 417.0 & 450.7 & 482.3 & 619.0 & 672.6 & 750.6 \\
\hline (Midpoint) & 272.7 & 313.3 & 346.8 & 369.7 & 484.5 & 529.1 & 582.8 \\
\hline \multicolumn{8}{|c|}{ Categories VIII and IX } \\
\hline Minimum & 207.7 & 247.6 & 281.1 & 298.5 & 399.4 & 438.4 & 491.3 \\
\hline Maximum & 463.1 & 506.2 & 540.2 & 579.4 & 734.9 & 800.6 & 926.1 \\
\hline (Midpoint) & 335.4 & 376.9 & 410.7 & 439.0 & 567.2 & 619.5 & 708.7 \\
\hline \multicolumn{8}{|l|}{ Category X } \\
\hline Minimum & 216.9 & 258.3 & 291.9 & 310.1 & 413.0 & 455.7 & 511.0 \\
\hline Maximum & 588.6 & 632.4 & 666.7 & 716.6 & 898.9 & 975.3 & $1,198.9$ \\
\hline (Midpoint) & 402.8 & 445.4 & 479.3 & 513.4 & 656.0 & 715.5 & 855.0 \\
\hline
\end{tabular}

Sources: Ministry of Finance and Economy; and Ministry of Budget and Development of Autonomous Provinces. 
Table 27. Madagascar: Central Government Capital Expenditure, 1994-98 1/

\begin{tabular}{|c|c|c|c|c|c|}
\hline & 1994 & 1995 & 1996 & 1997 & $\begin{array}{r}1998 \\
\text { Est. }\end{array}$ \\
\hline & & \multicolumn{3}{|c|}{ (In percent of total government capital expenditures) } & . \\
\hline General public services & 19.9 & 14.6 & 23.5 & 25.0 & 14.0 \\
\hline Education & 4.4 & 9.9 & 7.2 & 6.4 & 7.0 \\
\hline Health & 4,5 & 6.6 & 9.2 & 5.5 & 7.0 \\
\hline Social and community services & 9.0 & 7.5 & 1.4 & 1.7 & 12.0 \\
\hline Economic services & 62.2 & 61.4 & 58.6 & 61.4 & 60.0 \\
\hline Agriculture 2/ & 21.4 & 17.5 & 23.6 & 24.9 & 19.0 \\
\hline Industry, energy, and mining & 7.3 & 5.9 & 5.2 & 5.6 & 10.0 \\
\hline Transportation & 4.8 & 3.5 & 2.3 & 1.2 & 4.0 \\
\hline Communication services & 3.3 & 2.6 & 0.0 & 0.1 & 0.0 \\
\hline Public works 3/ & 23.7 & 29.6 & 26.8 & 26.7 & 26.0 \\
\hline Trade & 0.2 & 0.1 & 0.0 & 1.4 & 1.0 \\
\hline Scientific research & 1.7 & 2.2 & 0.6 & 1.4 & 0.0 \\
\hline \multirow[t]{2}{*}{ Total } & 100.0 & 100.0 & 100.0 & 100.0 & 100.0 \\
\hline & & \multicolumn{3}{|c|}{ (In billions of Malagasy francs, unless otherwise indicated) } & \\
\hline Budgetary capital expenditure & 313.0 & 422.7 & 498.4 & 489.5 & 815.2 \\
\hline Domestically financed & 153.3 & 172.1 & 247.3 & 208.4 & 392.1 \\
\hline Foreign loans & 159.7 & 250.6 & 251.1 & 281.1 & 423.1 \\
\hline Extrabudgetary & 35.0 & 23.7 & 34.3 & 66.1 & 64.9 \\
\hline On-lending 4/ & 48.0 & 41.7 & 76.9 & 42.1 & 74.8 \\
\hline External grant & 235.0 & 361.5 & 567.3 & 578.8 & 640.1 \\
\hline Autofinancing & 1.8 & 0.5 & 2.4 & 0.0 & 17.4 \\
\hline Other $5 /$ & 3.0 & 0.3 & 0.2 & 0.0 & 0.0 \\
\hline Total & 635.8 & 850.4 & $1,179.5$ & $1,176.5$ & $1,612.4$ \\
\hline In percent of GDP & 7.0 & 6.3 & 7.3 & 6.5 & 7.9 \\
\hline
\end{tabular}

Sources: Ministry of Finance and Economy; Ministry of Budget and Development of Autonomous Provinces; and Fund staff estimates.

1/ On a payment order basis for domestically financed expenditure and on a cash basis for other components.

$2 /$ Including livestock, fishing, water, and forestry.

3/ Mainly roads, ports, bridges, and other infrastructure.

4/ Foreign loans on-lent by the central government to public enterprises for investment purposes.

5/ Budgets annexes. 
Table 28. Madagascar: Revenue and Expenditure of the National Social Security Fund, 1994-98 (In billions of Malagasy francs)

\begin{tabular}{|c|c|c|c|c|c|}
\hline & 1994 & 1995 & 1996 & 1997 & $\begin{array}{r}1998 \\
\text { Est. }\end{array}$ \\
\hline Revenue & 39.2 & 47.2 & 53.6 & $\cdots$ & ... \\
\hline Social security contributions & 31.1 & 38.9 & 43.7 & $\ldots$. & $\ldots$ \\
\hline Employees & 2.5 & 3.5 & 3.2 & $\ldots$ & .... \\
\hline Employers & 28.6 & 35.3 & 40.5 & $\ldots$ & $\ldots$. \\
\hline Interest revenue & 8.1 & 8.1 & 9.4 & $\ldots$. & $\ldots$ \\
\hline Other revenue & 0.0 & 0.3 & 0.5 & $\cdots$ & $\ldots$ \\
\hline Expenditure & 27.3 & 45.6 & 52.3 & $\cdots$ & .... \\
\hline Social benefits & 20.0 & 33.8 & 36.2 & $\cdots$ & $\ldots$ \\
\hline Current expenditure & 5.5 & 8.4 & 11.7 & $\ldots$. & $\ldots$ \\
\hline Salaries and wages & 3.2 & 4.8 & 5.6 & $\cdots$ & $\ldots$ \\
\hline Goods and services & 2.3 & 3.4 & 5.9 & $\cdots$ & $\ldots$ \\
\hline Subsidies and other transfers & 0.0 & 0.2 & 0.2 & $\cdots$ & $\ldots$ \\
\hline Capital expenditure & 1.7 & 3.4 & 4.4 & $\cdots$ & $\cdots$ \\
\hline Overall surplus/deficit (-) & 11.9 & 1.6 & 1.3 & $\cdots$ & $\cdots$ \\
\hline
\end{tabular}

Source: National Social Security Fund. 
Table 29. Madagascar: Monetary Survey, 1994-98

(In billions of Malagasy francs; end of period)

\begin{tabular}{|c|c|c|c|c|c|c|c|c|c|c|}
\hline & \multirow[t]{2}{*}{1994} & \multirow{2}{*}{$\frac{1995}{\text { December }}$} & \multirow[t]{2}{*}{1996} & \multicolumn{4}{|c|}{1997} & \multicolumn{3}{|c|}{1998} \\
\hline & & & & Mar. & Jun. & Sep. & Dec. & Mar. & Jun. & $\overline{\text { Sep. }}$ \\
\hline Net foreign assets & 373.7 & 616.3 & $1,175.3$ & $1,227.6$ & $1,526.3$ & $1,625.0$ & $1,771.1$ & $1,716.3$ & $1,632.4$ & $1,431.1$ \\
\hline Central bank (net) & -105.3 & 111.1 & 713.4 & 771.4 & 968.8 & $1,078.0$ & $1,105.3$ & $1,092.2$ & $1,001.9$ & 850.0 \\
\hline Commercial banks (net) & 479.0 & 505.2 & 461.9 & 456.2 & 557.5 & 547.0 & 665.8 & 624.0 & 630.5 & 581.2 \\
\hline Long-term foreign liabilities & -169.8 & -162.7 & -186.4 & -192.5 & -197.3 & -201.3 & -206.8 & -208.0 & -201.1 & -203.9 \\
\hline Net domestic assets & $2,135.5$ & $2,263.7$ & $2,220.8$ & $2,167.1$ & $2,090.3$ & $2,125.3$ & $2,281.9$ & $2,243.7$ & $2,376.9$ & $2,726.6$ \\
\hline Net credit to government & 837.1 & 811.3 & 784.5 & 804.5 & 596.9 & 562.4 & 680.1 & 763.4 & 791.5 & 903.7 \\
\hline Central bank & 710.5 & 725.8 & 687.4 & 555.1 & 351.8 & 343.9 & 387.7 & 406.3 & 479.1 & 654.6 \\
\hline Commercial banks & 107.9 & 49.3 & 43.0 & 179.9 & 176.6 & 141.8 & 196.6 & 259.8 & 206.5 & 148.1 \\
\hline Other & 18.7 & 36.1 & 54.2 & 69.5 & 68.5 & 76.8 & 95.8 & 97.4 & 105.9 & 100.9 \\
\hline Credit to the economy & $1,356.5$ & $1,563.7$ & $1,599.4$ & $1,564.0$ & $1,619.0$ & $1,750.3$ & $1,814.1$ & $1,751.5$ & $1,834.4$ & $2,070.0$ \\
\hline Central bank & 11.6 & 12.6 & 14.1 & 14.2 & 15.0 & 15.1 & 15.1 & 15.1 & 15.3 & 15.4 \\
\hline Commercial banks & $1,334.7$ & $1,540.8$ & $1,572.0$ & $1,542.7$ & $1,595.0$ & $1,732.9$ & $1,797.6$ & $1,727.2$ & $1,813.9$ & $2,045.4$ \\
\hline Other & 10.2 & 10.3 & 13.3 & 7.1 & 9.0 & 2.3 & 1.4 & 9.2 & 5.3 & 9.2 \\
\hline Other items (net) & -58.0 & -111.2 & -163.1 & -201.4 & -125.6 & -187.5 & -212.3 & -271.2 & -249.0 & -247.0 \\
\hline Valuation adjustments, losses $(+)$ & 309.4 & 356.0 & 362.5 & 300.5 & 274.6 & 259.5 & 240.3 & 213.7 & 213.0 & 248.5 \\
\hline Other & -367.4 & -467.3 & -525.6 & -501.9 & -400.2 & -447.0 & -452.7 & -484.9 & -462.0 & -495.5 \\
\hline Broad money (M3) & $2,339.4$ & $2,717.3$ & $3,209.7$ & $3,202.1$ & $3,419.3$ & $3,548.9$ & $3,846.2$ & $3,751.9$ & $3,808.3$ & $3,953.8$ \\
\hline Currency & 614.5 & 758.7 & 831.2 & 792.6 & 846.8 & 929.3 & $1,025.6$ & 974.6 & $1,005.8$ & $1,117.1$ \\
\hline Demand deposits & 986.5 & $1,083.6$ & $1,349.5$ & $1,351.7$ & $1,482.2$ & $1,567.9$ & $1,658.6$ & $1,619.7$ & $1,727.2$ & $1,725.8$ \\
\hline Quasi money & 738.3 & 875.0 & $1,029.0$ & $1,057.8$ & $1,090.3$ & $1,051.7$ & $1,161.9$ & $1,157.6$ & $1,075.3$ & $1,110.9$ \\
\hline \multicolumn{11}{|l|}{ Of which } \\
\hline Residents' deposits in foreign currency & 230.7 & 284.6 & 285.1 & 299.4 & 377.2 & 403.1 & 441.9 & 434.7 & 465.0 & 446.7 \\
\hline Short-term bonds & 42.6 & 59.4 & 95.5 & 102.0 & 84.9 & 74.3 & 70.4 & 98.3 & 104.4 & 135.2 \\
\hline
\end{tabular}

Source: Central Bank of Madagascar. 
Table 30. Madagascar: Summary Accounts of the Central Bank, 1994-98

(In billions of Malagasy francs; end of period)

\begin{tabular}{|c|c|c|c|c|c|c|c|c|c|c|}
\hline & \multirow[t]{2}{*}{1994} & \multirow{2}{*}{$\frac{1995}{\text { December }}$} & \multirow[t]{2}{*}{1996} & \multicolumn{4}{|c|}{1997} & \multicolumn{3}{|c|}{1998} \\
\hline & & & & Mar. & Jun. & Sep. & Dec. & Mar. & Jun. & Sep. \\
\hline Net foreign assets & -105.3 & 111.1 & 713.4 & 771.4 & 968.8 & $1,078.0$ & $1,105.3$ & $1,092.2$ & $1,001.9$ & 850.0 \\
\hline Foreign assets & 241.4 & 375.0 & $1,043.4$ & $1,109.9$ & $1,297.2$ & $1,491.3$ & $1,492.7$ & $1,477.7$ & $1,353.1$ & $1,184.6$ \\
\hline Foreign liabilities (short term) & -17.8 & -15.3 & -14.2 & -13.0 & -12.6 & -15.3 & -18.8 & -14.0 & -14.7 & -9.7 \\
\hline Enhanced Structural Adjustment Facility & -328.9 & -248.6 & -315.8 & -325.5 & -315.8 & -398.1 & -368.6 & -371.4 & -336.5 & -324.9 \\
\hline Long-term foreign liabilities & -148.6 & -136.6 & -161.5 & -168.5 & -172.0 & -174.8 & -173.5 & -175.6 & -175.7 & -179.2 \\
\hline Net domestic assets & $1,163.3$ & $1,167.0$ & $1,022.9$ & 930.8 & 726.7 & 704.5 & 768.3 & 732.6 & 831.7 & $1,121.3$ \\
\hline Claims on government (net) & 710.5 & 725.8 & 687.4 & 555.1 & 351.8 & 343.9 & 387.7 & 406.3 & 479.1 & 654.6 \\
\hline Credit to government & $1,205.8$ & $1,278.6$ & $1,294.9$ & $1,126.0$ & $1,121.0$ & $1,186.7$ & $1,253.8$ & $1,154.3$ & $1,179.0$ & $1,510.4$ \\
\hline Statutory advances & 75.0 & 112.3 & 162.2 & 24.9 & 20.0 & 78.8 & 143.7 & 53.7 & 43.1 & 60.4 \\
\hline Consolidated loans & $1,103.0$ & $1,099.6$ & $1,099.6$ & $1,099.6$ & $1,099.6$ & $1,099.6$ & $1,099.6$ & $1,099.6$ & $1,099.6$ & $1,099.6$ \\
\hline Treasury bills & 0.0 & 0.0 & 0.0 & 0.0 & 0.0 & 0.0 & 0.0 & 0.0 & 31.5 & 82.2 \\
\hline Other & 27.8 & 66.7 & 33.0 & 1.5 & 1.4 & 8.3 & 10.5 & 1.0 & 4.7 & 268.2 \\
\hline Government deposits & 495.3 & 552.8 & 607.5 & 570.9 & 769.3 & 842.8 & 866.1 & 748.1 & 699.9 & 855.7 \\
\hline Of which: counterpart funds & 236.2 & 238.8 & 269.6 & 264.7 & 321.9 & 317.4 & 392.8 & 361.5 & 352.8 & 318.2 \\
\hline Claims on the economy & 11.6 & 12.6 & 14.1 & 14.2 & 15.0 & 15.1 & 15.1 & 15.1 & 15.3 & 15.4 \\
\hline Net claims on banks & 134.7 & 150.1 & -24.2 & 26.9 & 22.0 & 19.5 & 57.3 & 41.1 & 56.5 & 112.1 \\
\hline Claims on banks & 134.7 & 175.1 & 127.5 & 126.9 & 122.0 & 119.5 & 107.3 & 116.1 & 104.5 & 112.1 \\
\hline Reverse auction & 0.0 & -25.0 & -151.7 & -100.0 & -100.0 & -100.0 & -50.0 & -75.0 & -48.0 & 0.0 \\
\hline Other items (net) & 306.6 & 278.5 & 345.6 & 334.6 & 337.8 & 325.9 & 308.2 & 270.0 & 280.9 & 339.1 \\
\hline Valuation adjustments, losses (+) & 311.1 & 345.8 & 355.6 & 302.3 & 271.9 & 257.5 & 248.7 & 215.9 & 215.3 & 251.8 \\
\hline Operating losses $(+)$ & -13.6 & -2.6 & 16.9 & 14.4 & 9.6 & 11.7 & 47.0 & -2.7 & 3.7 & -1.2 \\
\hline Other & 9.1 & -64.7 & -26.9 & 17.9 & 56.3 & 56.7 & 12.4 & 56.8 & 61.9 & 88.4 \\
\hline Reserve money & 893.9 & $1,133.9$ & $1,573.3$ & $1,533.7$ & $1,521.4$ & $1,601.4$ & $1,697.1$ & $1,646.3$ & $1,637.3$ & $1,783.5$ \\
\hline Currency outside banks & 614.5 & 758.7 & 831.2 & 792.6 & 846.8 & 929.3 & $1,025.6$ & 974.6 & $1,005.8$ & $1,117.1$ \\
\hline Currency in banks & 18.5 & 26.5 & 29.5 & 29.4 & 33.2 & 27.4 & 26.3 & 25.9 & 38.4 & 30.5 \\
\hline Bank deposits & 260.8 & 348.7 & 712.6 & 711.6 & 641.4 & 644.7 & 645.3 & 645.8 & 593.1 & 635.8 \\
\hline Other demand deposits & 0.0 & 0.0 & 0.0 & 0.0 & 0.0 & 0.0 & 0.0 & 0.9 & 11.7 & 3.9 \\
\hline Other term deposits & 0.0 & 0.0 & 0.0 & 0.0 & 0.0 & 0.0 & 0.0 & 0.0 & 2.0 & 0.0 \\
\hline Residents' deposits in foreign currency & 15.5 & 7.5 & 1.5 & 0.0 & 2.1 & 6.2 & 3.0 & 2.0 & 6.9 & 4.7 \\
\hline
\end{tabular}

Source: Central Bank of Madagascar. 
Table 31. Madagascar: Foreign Reserve Assets and Liabilities of the Central Bank, 1994-98

(In millions of SDRs; end of period)

\begin{tabular}{|c|c|c|c|c|c|c|c|c|c|c|}
\hline & \multirow[t]{2}{*}{1994} & \multirow{2}{*}{$\frac{1995}{\text { December }}$} & \multirow[t]{2}{*}{1996} & \multicolumn{4}{|c|}{1997} & \multicolumn{3}{|c|}{1998} \\
\hline & & & & Mar. & Jun. & Sep. & Dec. & Mar. & Jun. & $\overline{\text { Sep. }}$ \\
\hline Foreign assets & 42.5 & 73.7 & 167.6 & 164.4 & 182.2 & 207.2 & 208.4 & 199.7 & 186.3 & 164.3 \\
\hline Of which: time deposits & 11.1 & 43.4 & 112.2 & 139.3 & 134.7 & 145.1 & 6.6 & 6.6 & 11.3 & 78.5 \\
\hline Foreign liabilities & 62.4 & 52.3 & 53.0 & 50.1 & 46.1 & 57.4 & 54.1 & 52.1 & 48.4 & 46.4 \\
\hline Nonresident deposits & 1.7 & 2.6 & 2.3 & 1.9 & 1.6 & 2.0 & 2.4 & 1.8 & 1.8 & 1.3 \\
\hline International organizations & 0.2 & 0.0 & 0.0 & 0.0 & 0.2 & 0.2 & 0.3 & 0.1 & 0.2 & 0.1 \\
\hline $\mathrm{IMF}$ & 59.2 & 49.3 & 50.7 & 48.2 & 44.4 & 55.3 & 51.5 & 50.2 & 46.3 & 45.1 \\
\hline External payments arrears & 1.3 & 0.4 & 0.0 & 0.0 & 0.0 & 0.0 & 0.0 & 0.0 & 0.0 & 0.0 \\
\hline Net foreign assets & -19.8 & 21.4 & 114.6 & 114.3 & 136.1 & 149.8 & 154.3 & 147.6 & 138.0 & 117.9 \\
\hline Excluding IMF and arrears & 40.7 & 71.1 & 165.4 & 162.5 & 180.5 & 205.1 & 205.8 & 197.8 & 184.3 & 162.9 \\
\hline \multicolumn{11}{|l|}{ Memorandum item: } \\
\hline \multicolumn{11}{|l|}{ Exchange rate: Malagasy franc } \\
\hline per SDR (end of period) & $5,675.3$ & $5,088.2$ & $6,224.2$ & $6,751.0$ & $7,118.2$ & $7,195.9$ & $7,161.5$ & $7,400.3$ & $7,261.6$ & $7,210.6$ \\
\hline
\end{tabular}

Source: Central Bank of Madagascar. 
Table 32. Madagascar: Summary Accounts of the Commercial Banks, 1994-98

(In billions of Malagasy francs; end of period)

\begin{tabular}{|c|c|c|c|c|c|c|c|c|c|c|}
\hline & \multirow[t]{2}{*}{1994} & \multirow{2}{*}{$\frac{1995}{\text { December }}$} & \multirow[t]{2}{*}{1996} & \multicolumn{4}{|c|}{1997} & \multicolumn{3}{|c|}{1998} \\
\hline & & & & Mar. & Jun. & Sep. & $\overline{\text { Dec. }}$ & Mar. & Jun. & $\overline{\text { Sep. }}$ \\
\hline Reserves & 279.3 & 375.2 & 742.1 & 741.1 & 674.6 & 672.1 & 671.5 & 671.7 & 631.5 & 666.4 \\
\hline Cash & 18.5 & 26.5 & 29.5 & 29.4 & 33.2 & 27.4 & 26.3 & 25.9 & 38.4 & 30.5 \\
\hline Deposits at central bank & 260.8 & 348.7 & 712.6 & 711.6 & 641.4 & 644.7 & 645.3 & 645.8 & 593.1 & 635.8 \\
\hline Net foreign assets & 479.0 & 505.2 & 461.9 & 456.2 & 557.5 & 547.0 & 665.8 & 624.0 & 630.5 & 581.2 \\
\hline Claims on government (net) & 107.9 & 49.3 & 43.0 & 179.9 & 176.6 & 141.8 & 196.6 & 259.8 & 206.5 & 148.1 \\
\hline Credit to government & 250.6 & 174.0 & 206.3 & 360.2 & 342.5 & 299.9 & 356.5 & 436.4 & 375.1 & 267.6 \\
\hline Government deposits & 142.8 & 124.8 & 163.3 & 180.3 & 165.9 & 158.1 & 159.9 & 176.6 & 168.5 & 119.5 \\
\hline Claims on private sector and state enterprises & $1,334.7$ & $1,540.8$ & $1,572.0$ & $1,542.7$ & $1,595.0$ & $1,732.9$ & $1,797.6$ & $1,727.2$ & $1,813.9$ & $2,045.4$ \\
\hline Deposits & $1,637.8$ & $1,845.3$ & $2,214.0$ & $2,231.0$ & $2,408.1$ & $2,460.0$ & $2,650.1$ & $2,569.6$ & $2,566.2$ & $2,582.9$ \\
\hline Demand deposits & 981.9 & $1,074.7$ & $1,338.3$ & $1,339.3$ & $1,469.8$ & $1,553.9$ & $1,643.7$ & $1,603.0$ & $1,695.0$ & $1,702.6$ \\
\hline Time deposits & 440.7 & 493.4 & 592.1 & 592.2 & 563.2 & 509.2 & 567.4 & 533.9 & 413.1 & 438.3 \\
\hline Foreign exchange deposits & 215.2 & 277.1 & 283.6 & 299.4 & 375.1 & 396.8 & 439.0 & 432.7 & 458.1 & 442.0 \\
\hline Short-term bonds & 42.6 & 59.4 & 95.5 & 102.0 & 84.9 & 74.3 & 70.4 & 98.3 & 104.4 & 135.2 \\
\hline Net recourse to the central bank & 134.7 & 150.1 & -24.2 & 26.9 & 22.0 & 19.5 & 57.3 & 41.1 & 56.5 & 112.1 \\
\hline Long-term foreign liabilities & 21.2 & 26.0 & 24.9 & 24.1 & 25.3 & 26.6 & 33.3 & 32.4 & 25.4 & 24.7 \\
\hline Net capital & 302.3 & 389.6 & 535.9 & 559.1 & 665.6 & 666.3 & 645.9 & 698.0 & 724.4 & 650.0 \\
\hline Other items (net) & 62.3 & 0.1 & -27.2 & -23.1 & -202.2 & -152.9 & -125.3 & -156.8 & -194.5 & -63.9 \\
\hline Currency gains/losses & 1.7 & -10.2 & -6.9 & 1.8 & .2 .7 & -2.0 & 8.4 & 2.3 & 2.3 & 3.3 \\
\hline Other & 60.6 & 10.3 & -20.3 & -24.9 & -199.4 & -150.9 & -133.7 & -159.1 & -196.8 & -67.3 \\
\hline \multicolumn{11}{|l|}{ Memorandum items: } \\
\hline Custom duty bills & 10.2 & 10.3 & 13.3 & 7.1 & 9.0 & 2.3 & 1.4 & 9.2 & 5.3 & 9.2 \\
\hline Post office checking deposits & 4.6 & 8.9 & 11.2 & 12.4 & 12.4 & 13.9 & 15.0 & 15.8 & 20.4 & 19.3 \\
\hline \multicolumn{11}{|l|}{ Private sector savings deposits with } \\
\hline the National Savings Fund & 24.3 & 37.5 & 56.3 & 64.1 & 65.1 & 65.1 & 82.2 & 90.7 & 90.7 & 90.7 \\
\hline
\end{tabular}

Source: Central Bank of Madagascar. 
Table 33. Madagascar: Structure of Market Interest Rates, 1991-98

(In percent per annum)

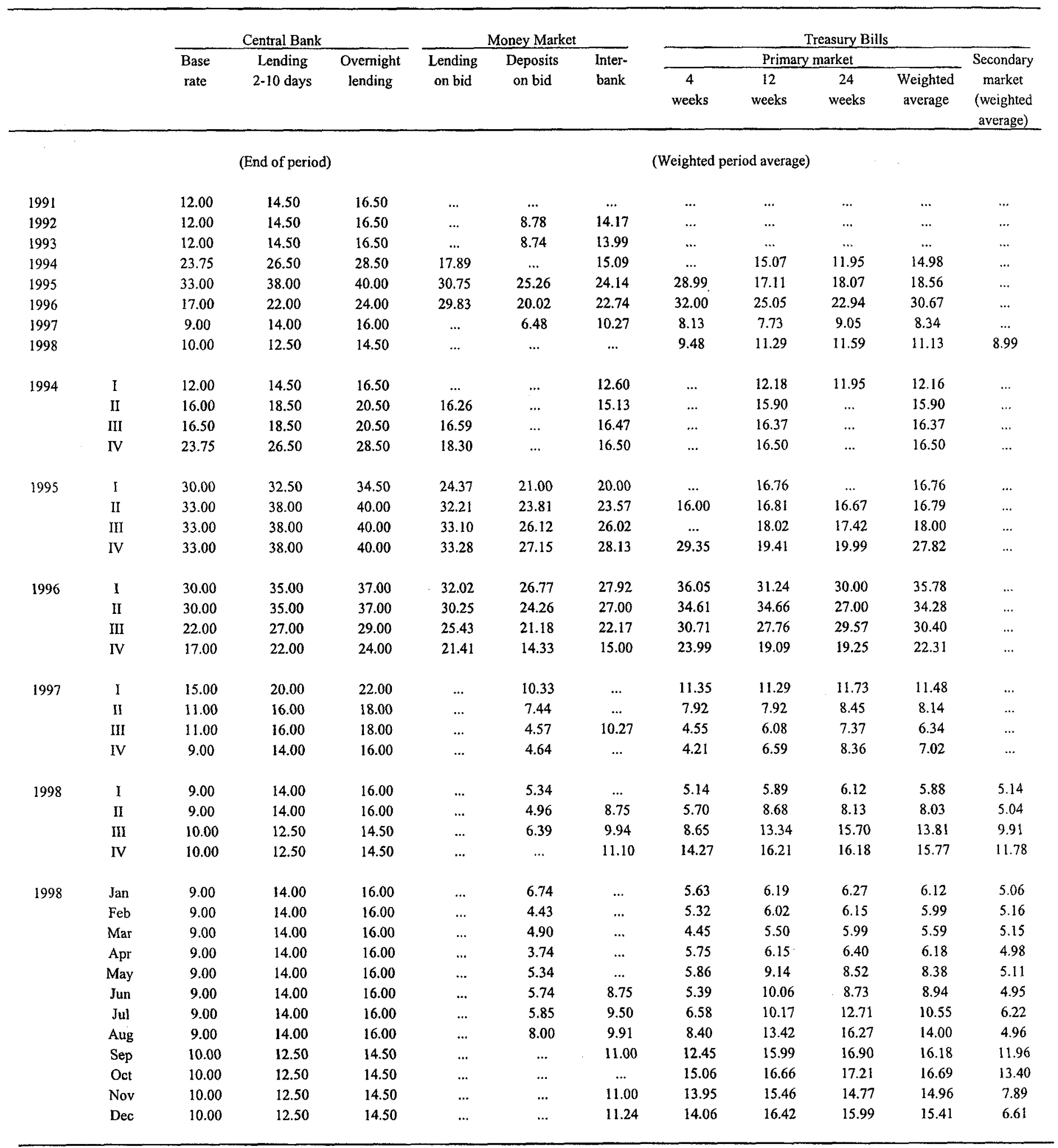

Source: Central Bank of Madagascar. 
Table 34. Madagascar: Structure of Commercial Banks' Interest Rates, 1991-98 1/

(In percent per annum)

\begin{tabular}{|c|c|c|c|c|c|c|c|c|c|c|}
\hline & & \multirow{2}{*}{$\begin{array}{l}\text { Base } \\
\text { Rate }\end{array}$} & \multicolumn{2}{|c|}{ Lending Rates } & \multicolumn{2}{|c|}{ Deposit Rates } & \multicolumn{2}{|c|}{ Lending Rates } & \multicolumn{2}{|c|}{ Deposit Rates } \\
\hline & & & $\begin{array}{l}\text { Short } \\
\text { term }\end{array}$ & $\begin{array}{c}\text { Medium } \\
\text { term }\end{array}$ & $\begin{array}{c}\text { Time \& } \\
>\text { one year }\end{array}$ & $\begin{array}{l}\text { Foreign } \\
\text { currency }\end{array}$ & $\begin{array}{l}\text { Short } \\
\text { term }\end{array}$ & $\begin{array}{c}\text { Medium } \\
\text { term }\end{array}$ & $\begin{array}{c}\text { Time \& } \\
>\text { one year }\end{array}$ & $\begin{array}{l}\text { Foreign } \\
\text { currency }\end{array}$ \\
\hline & & \multicolumn{5}{|c|}{ (Midpoint of the range; end of period) } & \multicolumn{4}{|c|}{ (Midpoint of weighted averages) $2 /$} \\
\hline 1991 & & 15.25 & $\ldots$ & $\ldots$ & $\ldots$ & $\ldots$ & $\ldots$ & $\ldots$ & $\ldots$ & $\ldots$ \\
\hline 1992 & & 15.59 & 16.75 & 15.13 & 13.00 & $\ldots$ & 15.82 & 16.34 & 13.81 & $\ldots$ \\
\hline 1993 & & 14.25 & 17.75 & 16.88 & 12.25 & $\ldots$ & 16.46 & 16.50 & 13.22 & $\ldots$ \\
\hline 1994 & & 19.00 & 20.50 & 21.25 & 12.00 & 3.29 & 19.64 & 18.13 & 13.15 & 2.22 \\
\hline 1995 & & 24.11 & 26.00 & 22.50 & 11.75 & 3.85 & 21.20 & 20.24 & 12.61 & 2.88 \\
\hline 1996 & & 19.73 & 23.25 & 20.75 & 12.00 & 2.89 & 21.90 & 22.95 & 13.56 & 2.39 \\
\hline 1997 & & 14.01 & 19.00 & 17.90 & 7.25 & 2.68 & 19.90 & 20.85 & 19.36 & 17.87 \\
\hline \multirow[t]{4}{*}{1995} & I & 20.68 & 22.40 & 19.50 & 10.13 & 1.38 & 21.01 & 20.15 & 10.64 & 1.25 \\
\hline & II & 21.98 & 21.00 & 22.75 & 12.38 & 3.00 & 20.14 & 17.81 & 13.75 & 4.40 \\
\hline & III & 22.77 & 24.75 & 22.75 & 12.13 & 3.13 & 21.51 & 20.41 & 12.61 & 2.37 \\
\hline & IV & 24.11 & 26.00 & 22.50 & 11.75 & 3.85 & 22.16 & 22.58 & 13.44 & 3.51 \\
\hline \multirow[t]{4}{*}{1996} & I & 24.49 & 25.75 & 24.50 & 14.50 & 3.25 & 22.45 & 22.77 & 14.22 & 2.17 \\
\hline & II & 24.00 & 25.38 & 23.63 & 14.50 & 2.14 & 23.67 & 24.03 & 14.53 & 1.58 \\
\hline & III & 24.53 & 25.25 & 23.63 & 12.13 & 2.25 & 21.61 & 23.87 & 13.50 & 2.17 \\
\hline & IV & 19.73 & 23.25 & 20.75 & 12.00 & 2.89 & 19.88 & 21.14 & 12.00 & 3.66 \\
\hline \multirow[t]{4}{*}{1997} & I & 17.50 & 19.50 & 21.40 & 9.26 & 1.75 & 21.64 & 20.68 & 10.17 & 1.08 \\
\hline & II & 15.50 & 19.50 & 21.40 & 9.13 & 2.50 & 20.39 & 21.88 & 10.07 & 1.04 \\
\hline & III & 16.00 & 20.00 & 19.15 & 7.50 & 2.50 & 19.13 & 17.28 & 9.48 & 1.06 \\
\hline & IV & 14.01 & 19.00 & 17.90 & 7.25 & 2.68 & 18.47 & 17.62 & 9.03 & 1.10 \\
\hline \multirow[t]{3}{*}{1998} & I & 12.50 & 18.50 & 16.38 & 5.50 & 2.50 & 18.35 & 15.54 & 8.78 & 1.07 \\
\hline & II & 12.50 & 18.13 & 16.13 & 4.25 & 2.50 & 17.37 & 14.92 & 9.54 & 1.23 \\
\hline & III & 12.50 & 18.25 & 16.13 & 4.25 & 2.50 & 17.38 & 15.36 & 9.12 & 1.33 \\
\hline
\end{tabular}

Source: Central Bank of Madagascar.

1/ Until 1994, the data are available only half-yearly

2/ Calculated by the banks on their outstandings; the midpoint is a simple average of the highest and lowest rates reported by the banks 
Table 35. Madagascar: Issues and Outstandings of Treasury Bills, 1993-98

(Nominal values; in billions of Malagasy francs, unless otherwise indicated)

\begin{tabular}{|c|c|c|c|c|c|c|c|c|c|c|c|c|c|c|c|c|c|}
\hline & & \multicolumn{7}{|c|}{ Total Issue } & \multicolumn{4}{|c|}{ Of Which: nonbanking sector 1/ } & \multicolumn{2}{|c|}{ Total Outstanding } & \multicolumn{3}{|c|}{ Of Which: nonbanking sector } \\
\hline & & \multicolumn{3}{|c|}{ Treasury preannouncement $2 /$} & \multirow{2}{*}{$\begin{array}{l}\begin{array}{c}\text { Total } \\
\text { bid }\end{array} \\
\text { (2) } \\
\end{array}$} & \multirow[b]{2}{*}{$\begin{array}{c}\text { Per- } \\
\text { centage } \\
(2) /(1)\end{array}$} & \multirow{2}{*}{$\begin{array}{l}\text { Bids } \\
\text { accepted } \\
\text { (3) } \\
\end{array}$} & \multirow[b]{2}{*}{$\begin{array}{c}\begin{array}{c}\text { Per- } \\
\text { centage }\end{array} \\
(3) /(2)\end{array}$} & \multirow{2}{*}{$\begin{array}{l}\text { Total } \\
\text { bid } \\
\text { (4) } \\
\end{array}$} & \multirow[b]{2}{*}{$\begin{array}{c}\text { Per- } \\
\text { centage } \\
(4) /(1 a)\end{array}$} & \multirow{2}{*}{$\begin{array}{c}\text { Bids } \\
\text { accepted } \\
(5) \\
\end{array}$} & \multirow[b]{2}{*}{$\begin{array}{c}\begin{array}{c}\text { Per- } \\
\text { centage }\end{array} \\
(5) /(4) \\
\end{array}$} & \multirow{2}{*}{$\begin{array}{c}\text { Net } \\
\text { purchases } \\
\text { (6) }\end{array}$} & \multirow{2}{*}{$\begin{array}{c}\text { Stock } \\
\text { at end of } \\
\text { period }\end{array}$} & \multirow{2}{*}{$\begin{array}{c}\begin{array}{c}\text { Net } \\
\text { purchases }\end{array} \\
\text { (8) }\end{array}$} & \multirow{2}{*}{$\begin{array}{c}\text { Stock } \\
\text { at end of } \\
\text { period } \\
(9) \\
\end{array}$} & \multirow{2}{*}{$\begin{array}{c}\text { Percentage } \\
\text { of } \\
\text { total } \\
(9) /(7) \\
\end{array}$} \\
\hline & & Total & $\begin{array}{l}\text { Of which: } \\
\text { competitive } \\
\text { market 3/ } \\
\text { (1a) }\end{array}$ & $\begin{array}{c}\text { Per- } \\
\text { centage } \\
(1 \mathrm{a}) /(1)\end{array}$ & & & & & & & & & & & & & \\
\hline 1993 & & $\ldots$ & $\ldots$ & $\ldots$ & 370.4 & $\ldots$ & 223.8 & 60.4 & $\ldots$ & $\ldots$ & $\ldots$ & $\ldots$ & 223.8 & 223.8 & $\ldots$ & $\ldots$ & $\ldots$ \\
\hline 1994 & & ... & $\ldots$ & $\ldots$ & 812.8 & $\ldots$ & 717.7 & 88.3 & $\ldots$ & $\ldots$ & $\ldots$ & $\ldots$ & 35.9 & 259.7 & $\ldots$ & $\ldots$ & $\ldots$ \\
\hline 1995 & & $\ldots$ & $\ldots$ & $\ldots$ & 896.1 & $\ldots$ & 824.5 & 92.0 & $\ldots$ & $\ldots$ & $\ldots$ & $\ldots$ & -78.8 & 180.9 & 31.5 & 31.5 & 17.4 \\
\hline 1996 & & $\ldots$ & $\ldots$ & $\ldots$ & $1,694.5$ & $\ldots$ & $1,387.6$ & 81.9 & $\ldots$ & $\ldots$ & $\ldots$ & $\ldots$ & 95.7 & 276.6 & 47.7 & 79.2 & 28.6 \\
\hline 1997 & & $1,099.5$ & $1,059.5$ & 96.4 & $2,909.5$ & $\ldots$ & $1,650.0$ & 56.7 & 286.1 & 27.0 & 177.2 & 61.9 & 198.4 & 475.0 & 49.1 & 128.3 & 27.0 \\
\hline 1998 & & $2,330.0$ & $1,925.0$ & 82.6 & $2,899.0$ & $\ldots$ & $1,953.2$ & 67.4 & 845.5 & 43.9 & 632.9 & 74.9 & 156.1 & 631.1 & 163.0 & 291.3 & 46.2 \\
\hline \multirow[t]{4}{*}{1995} & 1 & $\ldots$ & $\ldots$ & $\ldots$ & 260.9 & $\ldots$ & 199.3 & 76.4 & $\ldots$ & $\ldots$ & $\ldots$ & $\ldots$ & 4.4 & 264.1 & 1.1 & 1.1 & 0.4 \\
\hline & II & $\ldots$ & $\ldots$ & $\cdots$ & 226.0 & $\cdots$ & 220.2 & 97.4 & $\ldots$ & $\ldots$ & $\ldots$ & $\ldots$ & 21.5 & 285.6 & 28.5 & 29.6 & 10.4 \\
\hline & III & $\ldots$ & $\ldots$ & $\ldots$ & 148.0 & $\ldots$ & 144.0 & 97.3 & $\ldots$ & $\ldots$ & $\ldots$ & $\ldots$ & -61.8 & 223.8 & 3.1 & 32.7 & 14.6 \\
\hline & IV & $\ldots$ & $\ldots$ & $\ldots$ & 261.2 & $\cdots$ & 261.0 & 99.9 & $\ldots$ & $\ldots$ & $\ldots$ & $\ldots$ & -42.9 & 180.9 & -1.2 & 31.5 & 17.4 \\
\hline \multirow[t]{4}{*}{1996} & I & $\ldots$ & $\ldots$ & $\ldots$ & 277.1 & $\ldots$ & 277.1 & 100.0 & $\ldots$ & $\ldots$ & $\ldots$ & $\ldots$ & 10.1 & 191.0 & 4.9 & 36.4 & 19.1 \\
\hline & II & $\ldots$ & $\ldots$ & $\ldots$ & 312.6 & $\ldots$ & 312.6 & 100.0 & $\ldots$ & $\ldots$ & $\ldots$ & $\ldots$ & 3.9 & 194.9 & 2.9 & 39.3 & 20.2 \\
\hline & III & $\ldots$ & $\ldots$ & $\ldots$ & 392.8 & $\ldots$ & 392.5 & 99.9 & $\ldots$ & $\ldots$ & $\ldots$ & $\ldots$ & 23.2 & 218.1 & 23.1 & 62.4 & 28.6 \\
\hline & IV & $\ldots$ & $\ldots$ & $\ldots$ & 712.0 & $\ldots$ & 405.4 & 56.9 & $\ldots$ & $\ldots$ & $\ldots$ & $\ldots$ & 58.5 & 276.6 & 16.8 & 79.2 & 28.6 \\
\hline \multirow[t]{4}{*}{1997} & I & $\ldots$ & $\ldots$ & $\ldots$ & 675.0 & $\ldots$ & 502.8 & 74.5 & $\ldots$ & $\ldots$ & $\ldots$ & $\ldots$ & 178.7 & 455.3 & 18.4 & 97.6 & 21.4 \\
\hline & II & 252.0 & 252.0 & 100.0 & 908.1 & $\ldots$ & 408.7 & 45.0 & $\ldots$ & $\ldots$ & $\ldots$ & $\ldots$ & -1.6 & 453.7 & 15.3 & 112.9 & 24.9 \\
\hline & III & 352.5 & 352.5 & 100.0 & 496.3 & 140.8 & 273.5 & 55.1 & 94.8 & 26.9 & 67.9 & 71.6 & -70.0 & 383.7 & -18.5 & 94.4 & 24.6 \\
\hline & IV & 495.0 & 455.0 & 91.9 & 830.0 & 167.7 & 465.0 & 56.0 & 135.1 & 29.7 & 71.4 & 52.9 & 91.3 & 475.0 & 33.9 & 128.3 & 27.0 \\
\hline \multirow[t]{4}{*}{1998} & I & 475.0 & 350.0 & 73.7 & 853.1 & 179.6 & 420.0 & 49.2 & 148.7 & 42.5 & 71.2 & 47.9 & 73.0 & 548.0 & -7.4 & 120.9 & 22.1 \\
\hline & II & 590.0 & 490.0 & 83.1 & 777.5 & 131.8 & 514.8 & 66.2 & 204.0 & 41.6 & 164.9 & 80.8 & 38.7 & 586.7 & 63.8 & 184.7 & 31.5 \\
\hline & III & 600.0 & 480.0 & 80.0 & 574.9 & 95.8 & 472.4 & 82.2 & 180.5 & 37.6 & 167.6 & 92.9 & 44.4 & 631.1 & 87.8 & 272.5 & 43.2 \\
\hline & IV & 665.0 & 605.0 & 91.0 & 693.5 & 104.3 & 546.0 & 78.7 & 312.3 & 51.6 & 229.2 & 73.4 & 0.0 & 631.1 & 18.8 & 291.3 & 46.2 \\
\hline 1998 & Jan & 125.0 & 85.0 & 68.0 & 227.4 & 181.9 & 110.0 & 48.4 & 47.4 & 55.8 & 23.1 & 48.7 & -12.0 & 462.9 & -20.3 & 108.0 & 23.3 \\
\hline & Feb & 160.0 & 110.0 & 68.8 & 425.6 & 266.0 & 160.0 & 37.6 & 49.5 & 45.0 & 22.1 & 44.6 & 60.0 & 522.9 & 8.9 & 116.9 & 22.3 \\
\hline & Mar & 190.0 & 155.0 & 81.6 & 200.1 & 105.3 & 150.0 & 75.0 & 51.8 & 33.4 & 26.0 & 50.2 & 25.0 & 547.9 & 4.1 & 120.9 & 22.1 \\
\hline & Apr & 160.0 & 140.0 & 87.5 & 132.2 & 82.6 & 122.6 & 92.7 & 38.2 & 27.3 & 35.6 & 93.2 & 8.6 & 556.5 & 18.9 & 139.8 & 25.1 \\
\hline & May & 260.0 & 210.0 & 80.8 & 381.4 & 146.7 & 237.0 & 62.1 & 97.9 & 46.6 & 81.3 & 83.0 & 2.2 & 558.7 & $37.3^{\circ}$ & 177.2 & 31.7 \\
\hline & Jun & 170.0 & 140.0 & 82.4 & 263.9 & 155.2 & 155.2 & 58.8 & 67.9 & 48.5 & 48.0 & 70.7 & 27.9 & 586.6 & 7.6 & 184.7 & 31.5 \\
\hline & Jul & 180.0 & 160.0 & 88.9 & 136.8 & 76.0 & 131.6 & 96.2 & 50.9 & 31.8 & 47.8 & 93.9 & 14.1 & 600.7 & 24.1 & 208.8 & 34.8 \\
\hline & Aug & 195.0 & 165.0 & 84.6 & 201.1 & 103.1 & 172.8 & 85.9 & 50.6 & 30.7 & 46.8 & 92.5 & -3.2 & 597.5 & 33.8 & 242.6 & 40.6 \\
\hline & Sep & 225.0 & 155.0 & 68.9 & 237.0 & 105.3 & 168.0 & 70.9 & 79.0 & 51.0 & 73.0 & 92.4 & 33.5 & 631.0 & 29.9 & 272.5 & 43.2 \\
\hline & Oct & 240.0 & 210.0 & 87.5 & 274.7 & 114.5 & 209.1 & 76.1 & 111.5 & 53.1 & 64.1 & 57.5 & -13.2 & 617.8 & -35.9 & 236.6 & 38.3 \\
\hline & Nov & 220.0 & 200.0 & 90.9 & 192.6 & 87.5 & 154.6 & 80.3 & 107.1 & 53.6 & 88.5 & 82.7 & 3.1 & 620.9 & 59.0 & 295.6 & 47.6 \\
\hline & Dec & 205.0 & 195.0 & 95.1 & 226.2 & 110.3 & 182.3 & 80.6 & 93.7 & 48.1 & 76.6 & 81.8 & 10.1 & 631.0 & -4.3 & 291.3 & 46.2 \\
\hline
\end{tabular}

Source: Central Bank of Madagascar.

1/ Competitive market only, since May 16, 1997.

2/ Since May 16, 1997 only.

$3 /$ The noncompetitive segment of the market is open only to intermediaries in the secondary market; bills are sold at a price equal to the average of bids in the competitive auction market. 
Table 36. Madagascar: Profitability of Banks, 1994-98

(In millions of Malagasy francs)

\begin{tabular}{|c|c|c|c|c|c|}
\hline & 1994 & 1995 & 1996 & 1997 & $\begin{array}{c}1998 \\
\text { June }\end{array}$ \\
\hline 1. Margin on treasury and financial operations & 21,605 & $-24,327$ & 62,968 & 62,162 & 25,943 \\
\hline Revenue (including short-term placements) & 44,280 & 66,976 & 88,887 & 62,281 & 25,981 \\
\hline Cost (including refinancing and penalties) & 22,675 & 91,303 & 25,919 & 119 & 38 \\
\hline 2. Margin on operations with customers & 82,768 & 147,245 & 120,642 & 110,248 & 60,451 \\
\hline Revenue (interest received on loans) & 177,537 & 249,042 & 259,291 & 195,230 & 85,334 \\
\hline Cost (interest paid on deposits) & 94,769 & 101,797 & 138,649 & 84,982 & 24,883 \\
\hline 3. Margin on other operations & 164,164 & 145,539 & 114,743 & 121,036 & 59,939 \\
\hline Revenue & 180,530 & 173,116 & 138,447 & 164,967 & 129,161 \\
\hline Of which: revenue from foreign exchange operations & 124,562 & 66,401 & 65,189 & 85,108 & 87,443 \\
\hline Cost & 16,366 & 27,577 & 23,704 & 43,931 & 69,222 \\
\hline Of which: cost of foreign exchange operations & 10,404 & 23,962 & 12,889 & 27,705 & 62,023 \\
\hline 4. Net banking earnings $(1)+(2)+(3)$ & 268,537 & 268,457 & 298,353 & 293,446 & 146,333 \\
\hline 5. Other revenues & 3,465 & 6,170 & 7,811 & 8,321 & 4,640 \\
\hline 6. Total net banking earnings $(4)+(5)$ & 272,002 & 274,627 & 306,164 & 301,767 & 150,973 \\
\hline 7. Operating cost & 79,816 & 114,240 & 132,426 & 144,343 & 74,938 \\
\hline Staff & 38,545 & 57,020 & 67,119 & 72,975 & 38,116 \\
\hline Overhead & 41,271 & 57,220 & 65,308 & 71,368 & 36,822 \\
\hline 8. Amortization & 11,645 & 14,606 & 14,212 & 17,687 & 8,999 \\
\hline 9. Total costs $(7)+(8)$ & 91,461 & 128,846 & 146,638 & 162,030 & 83,936 \\
\hline 10. Net earnings balance (6)-(9) & 180,541 & 145,781 & 159,527 & 139,737 & 67,036 \\
\hline 11. Net provisions for nonperforming portfolios & 78,317 & 176,515 & 6,255 & 58,344 & $-1,018$ \\
\hline 12. Other provisions & 51,702 & 47,904 & 39,853 & $-12,371$ & $-3,369$ \\
\hline 13. Other profits and losses & $-6,771$ & 39,377 & 64 & $-6,730$ & $-2,064$ \\
\hline 14. Corporate tax & 41,415 & 44,493 & 53,298 & 43,700 & 19,820 \\
\hline 15. Net balance $(10)-(11)-(12)+(13)-(14)$ & 2,335 & $-83,753$ & 60,184 & 43,334 & 49,539 \\
\hline \multicolumn{6}{|l|}{ Memorandum item: } \\
\hline Ratio of operating costs to total earnings $(9) /(6)$ (in percent) & 33.6 & 46.9 & 47.9 & 53.7 & 55.6 \\
\hline
\end{tabular}

Source: Financial and Banking Supervision Commission (CSBF), Annual Reports. 
Table 37. Madagascar: Summary Balance of Payments, 1994-98

(In millions of SDRs, unless otherwise indicated)

\begin{tabular}{|c|c|c|c|c|c|}
\hline & 1994 & 1995 & 1996 & 1997 & $\begin{array}{l}1998 \\
\text { Prov. }\end{array}$ \\
\hline Trade balance & -68.6 & -69.4 & -83.4 & -128.8 & -108.7 \\
\hline Exports, f.o.b. & 312.4 & 344.6 & 360.6 & 366.7 & 385.2 \\
\hline Imports, f.o.b. & -381.0 & -414.0 & -444.0 & -495.6 & -493.9 \\
\hline Services (net) & -189.9 & -193.4 & -171.6 & -157.3 & -153.9 \\
\hline Service receipts & 145.1 & 162.1 & 210.7 & 200.3 & 211.6 \\
\hline Service payments & -335.0 & -355.5 & -382.2 & -357.6 & -365.5 \\
\hline Of which : investment income & -105.6 & -114.5 & -114.9 & -80.8 & -60.5 \\
\hline scheduled public interest & -99.9 & -108.2 & -111.1 & -71.8 & -51.6 \\
\hline Private unrequited transfers (net) & 32.1 & 49.5 & 60.7 & 84.2 & 60.0 \\
\hline \multicolumn{6}{|l|}{ Current account balance, excluding } \\
\hline & -226.5 & -213.3 & -194.3 & -202.0 & -202.6 \\
\hline Public unrequited transfers (net) & 81.0 & 67.0 & 91.0 & 140.2 & 92.2 \\
\hline Current grants & 30.9 & 11.2 & 22.6 & 57.8 & 6.4 \\
\hline Balance of payments grants & 8.9 & 1.7 & 8.5 & 45.7 & 3.0 \\
\hline Other current grants (net) $1 /$ & 22.0 & 9.5 & 14.1 & 12.1 & 3.4 \\
\hline Project grants & 50.1 & 55.8 & 68.4 & 82.4 & 85.8 \\
\hline \multicolumn{6}{|l|}{ Current account balance, including } \\
\hline net official transfers & -145.5 & -146.3 & -103.3 & -61.8 & -110.4 \\
\hline Capital account & -69.6 & -37.0 & 26.5 & 81.5 & 22.2 \\
\hline Public sector (net) & -106.6 & -93.1 & -50.9 & 75.0 & -16.2 \\
\hline Drawings & 52.3 & 54.9 & 60.0 & 152.3 & 73.7 \\
\hline Amortization & -155.5 & -142.8 & -104.9 & -72.6 & -89.9 \\
\hline Long-term liabilities (net) $2 /$ & -3.4 & -5.2 & -6.0 & -4.8 & 0.0 \\
\hline Private sector (net) & 37.0 & 56.1 & 77.4 & 6.5 & 38.4 \\
\hline Commercial banks (net) & -6.7 & -14.9 & 25.3 & -18.6 & 12.3 \\
\hline Direct investment & 4.0 & 6.4 & 7.0 & 10.0 & 20.5 \\
\hline Of which : privatization proceeds & 0.0 & 0.0 & 0.0 & 0.0 & 9.3 \\
\hline Other (including errors and omissions) & 39.7 & 64.6 & 45.1 & 15.2 & 5.6 \\
\hline Overall balance & -215.1 & -183.3 & -76.8 & 19.7 & -88.2 \\
\hline
\end{tabular}


Table 37. Madagascar: Summary Balance of Payments, 1994-98 (concluded)

(In millions of SDRs, unless otherwise indicated)

\begin{tabular}{|c|c|c|c|c|c|}
\hline & 1994 & 1995 & 1996 & 1997 & $\begin{array}{l}1998 \\
\text { Prov. }\end{array}$ \\
\hline Financing & 215.1 & 183.3 & 76.8 & -19.7 & 88.2 \\
\hline Net foreign assets (increase -) & 6.7 & -39.8 & -92.6 & -39.5 & 54.0 \\
\hline Use of Fund credit (net) & -8.2 & -9.4 & 1.9 & 0.7 & -10.3 \\
\hline Net repurchases & -4.5 & -0.6 & 0.0 & 0.0 & 0.0 \\
\hline Enhanced Structural Adjustment Facility (ESAF) disbursements & 0.0 & 0.0 & 13.6 & 13.6 & 0.0 \\
\hline ESAF repayments & -3.7 & -8.8 & -11.6 & -12.9 & -10.3 \\
\hline Change in other liabilities & -1.4 & 0.6 & -0.6 & 0.0 & 0.0 \\
\hline Change in gross assets (increase -) & 15.9 & -31.0 & -93.9 & -40.2 & 64.3 \\
\hline Change in central bank arrears (increase + ) & 0.4 & 0.0 & 0.0 & 0.0 & 0.0 \\
\hline Net change in arrears (excluding central bank) & 208.4 & 223.1 & 169.3 & -783.2 & -353.1 \\
\hline Cash payments and arrears accumulation & .. & .. &.. & -24.7 & -4.2 \\
\hline Debt rescheduling & .. &.. &.. & -758.5 & -348.9 \\
\hline Debt relief and cancellation & 0.0 & 0.0 & 0.0 & 803.0 & 387.3 \\
\hline Residual financing gap & 0.0 & 0.0 & 0.0 & 0.0 & 0.0 \\
\hline \multicolumn{6}{|l|}{ Memorandum items: } \\
\hline \multicolumn{6}{|l|}{ External current account / GDP (in percent) } \\
\hline Excluding net official transfers & -10.9 & -10.2 & -7.1 & -7.8 & -7.4 \\
\hline Including net official transfers & -7.0 & -7.0 & -3.7 & -2.4 & -4.0 \\
\hline Gross official reserves & 42.7 & 73.7 & 167.6 & 207.8 & 143.5 \\
\hline (in weeks of imports) & 3.6 & 5.9 & 12.3 & 14.0 & 9.3 \\
\hline \multicolumn{6}{|l|}{ Scheduled external debt service $3 / 4 /$} \\
\hline Before debt relief & 59.8 & 53.6 & 41.3 & 29.7 & 27.6 \\
\hline After debt relief & 59.8 & 53.6 & 41.3 & 21.8 & 20.3 \\
\hline \multicolumn{6}{|l|}{ Public sector scheduled debt service 5/ } \\
\hline Before debt relief & 153.7 & 147.1 & 91.7 & 58.2 & 48.6 \\
\hline After debt relief & 153.7 & 147.1 & 91.7 & 39.8 & 33.9 \\
\hline External debt stock (after debt relief) 4/ & $2,826.8$ & $2,933.2$ & $3,085.1$ & $3,104.7$ & $2,938.5$ \\
\hline In percent of GDP & 136.6 & 140.9 & 111.9 & 120.6 & 107.2 \\
\hline $\begin{array}{l}\text { In percent of exports of goods and } \\
\text { nonfactor services }\end{array}$ & 620.2 & 584.2 & 545.7 & 553.1 & 503.5 \\
\hline \multicolumn{6}{|l|}{ Annual percentage changes } \\
\hline Export volume & 7.7 & 9.3 & 18.6 & 7.5 & 7.0 \\
\hline Import volume & 2.0 & 6.8 & 0.6 & 14.7 & 11.5 \\
\hline Real GDP & 0.0 & 1.7 & 2.1 & 3.6 & $3: 9$ \\
\hline
\end{tabular}

Sources: Central Bank of Madagascar; and Fund staff estimates.

1/ Other official grants less payments due to scholarships and contributions to international organizations.

2/ Involves liabilities arising from Madagascar debts bought by certain organizations.

$3 /$ In percent of exports of goods and nonfactor services.

4/ Including obligations to the IMF; and takes into account flow reschedulings for 1997-98.

$5 /$ In percent of government revenue. 
Table 38. Madagascar: Composition of Exports, f.o.b., 1994-98

(Values in millions of SDRs; volumes in thousand tons; and unit values in SDR per kilogram)

\begin{tabular}{|c|c|c|c|c|c|}
\hline & 1994 & 1995 & 1996 & $\begin{array}{r}1997 \\
\text { Est. }\end{array}$ & $\begin{array}{l}1998 \\
\text { Prov. }\end{array}$ \\
\hline \multicolumn{6}{|l|}{ Coffee } \\
\hline Value & 59.9 & 61.2 & 42.6 & 23.9 & 31.3 \\
\hline Volume & 40.6 & 39.2 & 44.2 & 25.9 & 32.0 \\
\hline Unit price & 1.5 & 1.6 & 1.0 & 0.9 & 1.0 \\
\hline \multicolumn{6}{|l|}{ Vanilla } \\
\hline Value & 44.2 & 27.2 & 13.5 & 6.9 & 8.0 \\
\hline Volume & 1.1 & 0.8 & 1.2 & 0.7 & 0.5 \\
\hline Unit price & 41.5 & 36.3 & 11.6 & 10.7 & 17.4 \\
\hline \multicolumn{6}{|l|}{ Cloves } \\
\hline Value & 5.9 & 7.2 & 3.6 & 8.9 & 12.1 \\
\hline Volume & 17.1 & 17.1 & 7.1 & 15.8 & 16.9 \\
\hline Unit price & 0.3 & 0.4 & 0.5 & 0.6 & 0.7 \\
\hline \multicolumn{6}{|l|}{ Pepper } \\
\hline Value & 2.1 & 2.0 & 2.8 & 2.1 & 2.2 \\
\hline Volume & 2.3 & 1.4 & 1.9 & 0.9 & 0.7 \\
\hline Unit price & 0.9 & 1.4 & 1.5 & 2.3 & 3.1 \\
\hline \multicolumn{6}{|l|}{ Shellfish } \\
\hline Value & 40.4 & 38.8 & 43.6 & 46.8 & 49.6 \\
\hline Volume & 7.7 & 8.2 & 8.0 & 8.0 & 8.3 \\
\hline Unit price & 5.2 & 4.7 & 5.4 & 5.8 & 6.0 \\
\hline \multicolumn{6}{|l|}{ Sugar } \\
\hline Value & 6.9 & 9.4 & 11.5 & 8.2 & 8.2 \\
\hline Volume & 23.1 & 22.3 & 25.7 & 20.6 & 20.6 \\
\hline Unit price & 0.3 & 0.4 & 0.5 & 0.4 & 0.4 \\
\hline \multicolumn{6}{|l|}{ Meat } \\
\hline Value & 3.9 & 5.8 & 2.7 & 0.9 & 0.1 \\
\hline Volume & 2.8 & 3.8 & 1.8 & 0.6 & 0.1 \\
\hline Unit price & 1.4 & 1.5 & 1.6 & 1.4 & 0.8 \\
\hline \multicolumn{6}{|l|}{ Cocoa } \\
\hline Value & 1.9 & 2.5 & 2.3 & 0.6 & 0.9 \\
\hline Volume & 2.2 & 3.3 & 2.9 & 0.7 & 1.0 \\
\hline Unit price & 0.9 & 0.8 & 0.8 & 0.9 & 0.9 \\
\hline \multicolumn{6}{|l|}{ Cotton cloth } \\
\hline Value & 9.3 & 7.4 & 7.1 & 12.3 & 15.7 \\
\hline Volume & 2.4 & 2.0 & 1.5 & 2.5 & 4.0 \\
\hline Unit price & 3.8 & 3.7 & 4.8 & 5.0 & 3.9 \\
\hline
\end{tabular}


- $46-$

Table 38. Madagascar: Composition of Exports, f.o.b., 1994-98 (concluded)

(Values in millions of SDRs; volumes in thousand tons; and unit values in SDR per kilogram)

\begin{tabular}{|c|c|c|c|c|c|}
\hline & 1994 & 1995 & 1996 & $\begin{array}{r}1997 \\
\text { Est. }\end{array}$ & $\begin{array}{l}1998 \\
\text { Prov. }\end{array}$ \\
\hline \multicolumn{6}{|l|}{ Sisal } \\
\hline Value & 2.3 & 2.8 & 3.5 & 1.4 & 0.7 \\
\hline Volume & 10.4 & 11.2 & 11.0 & 3.8 & 1.6 \\
\hline Unit price & 0.2 & 0.3 & 0.3 & 0.4 & 0.4 \\
\hline \multicolumn{6}{|l|}{ Petroleum products } \\
\hline Value & 3.8 & 10.0 & 9.0 & 10.6 & 7.5 \\
\hline Volume & 83.1 & 185.1 & 121.8 & 162.9 & 150.3 \\
\hline Unit price & 0.0 & 0.1 & 0.1 & 0.1 & 0.1 \\
\hline \multicolumn{6}{|l|}{ Chromite } \\
\hline Value & 3.8 & 7.7 & 6.8 & 6.5 & 6.9 \\
\hline Volume & 90.0 & 128.5 & 110.1 & 117.8 & 138.4 \\
\hline Unit price & 0.0 & 0.1 & 0.1 & 0.1 & 0.1 \\
\hline \multicolumn{6}{|l|}{ Graphite } \\
\hline Value & 5.5 & 6.3 & 5.4 & 5.0 & 5.3 \\
\hline Volume & 15.6 & 16.5 & 14.3 & 14.0 & 14.3 \\
\hline Unit price & 0.4 & 0.4 & 0.4 & 0.4 & 0.4 \\
\hline \multicolumn{6}{|l|}{ Essence of cloves } \\
\hline Value & 2.2 & 3.7 & 2.9 & 3.8 & 2.7 \\
\hline Volume & 1.3 & 1.6 & 1.0 & 1.4 & 1.0 \\
\hline Unit price & 1.7 & 2.4 & 2.9 & 2.7 & 2.8 \\
\hline \multicolumn{6}{|l|}{ Rice } \\
\hline Value & 0.0 & 0.2 & 0.0 & 0.0 & 0.0 \\
\hline Volume & 0.0 & 0.0 & 0.0 & 0.0 & 0.0 \\
\hline Unit price & 0.0 & 0.0 & 0.0 & 0.0 & 0.0 \\
\hline \multicolumn{6}{|l|}{ Other items $1 /$} \\
\hline Value & 75.4 & 82.8 & 82.3 & 93.1 & 90.0 \\
\hline Volume & 0.6 & 0.6 & 0.7 & 0.9 & 0.9 \\
\hline Unit price & 119.8 & 140.5 & 110.9 & 106.2 & 95.8 \\
\hline \multicolumn{6}{|c|}{ Exports of the export processing zone (EPZ) $1 /$} \\
\hline Value & 44.7 & 69.7 & 120.9 & 135.8 & 143.9 \\
\hline Volume & 0.4 & 0.5 & 1.1 & 1.3 & 1.5 \\
\hline Unit price & 119.8 & 140.5 & 110.9 & 106.2 & 95.9 \\
\hline Total value & 312.4 & 344.6 & 360.6 & 366.7 & 385.2 \\
\hline (percentage change) & 31.3 & 10.3 & 4.6 & 1.7 & 5.0 \\
\hline \multicolumn{6}{|l|}{ Memorandum items: } \\
\hline Value of traditional exports $2 /$ & 112.1 & 97.5 & 62.5 & 41.8 & 53.6 \\
\hline In percent of total & 35.9 & 28.3 & 17.3 & 11.4 & 13.9 \\
\hline Value of non-traditional exports & 200.3 & 247.1 & 298.1 & 324.9 & 331.6 \\
\hline In percent of total & 64.1 & 71.7 & 82.7 & 88.6 & 86.1 \\
\hline Value of manufactured exports $3 /$ & 133.2 & 169.9 & 219.3 & 251.8 & 257.1 \\
\hline In percent of total & 42.6 & 49.3 & 60.8 & 68.7 & 66.7 \\
\hline
\end{tabular}

Sources: Central Bank of Madagascar; and Fund staff estimates.

1/In 1994 and 1995, EPZ exports include reexports. From 1996 onwards, reexports are included with other items.

$2 /$ Includes coffee, vanilla, cloves, and pepper.

$3 /$ Includes cotton cloth, petroleum, other items, and EPZ exports. 
Table 39. Madagascar: Composition of Imports, c.i.f, 1994-98

\begin{tabular}{|c|c|c|c|c|c|}
\hline & 1994 & 1995 & 1996 & $\begin{array}{r}1997 \\
\text { Est. }\end{array}$ & $\begin{array}{l}1998 \\
\text { Prov. }\end{array}$ \\
\hline & \multicolumn{5}{|c|}{ (In millions of SDRs) } \\
\hline Food & 55.0 & 44.1 & 41.5 & 35.3 & 40.8 \\
\hline Rice & 26.2 & 12.2 & 5.9 & 12.3 & 12.8 \\
\hline Other & 28.9 & 32.0 & 35.6 & 23.0 & 28.0 \\
\hline Nonfood consumer goods & 74.5 & 69.1 & 73.8 & 88.3 & 101.3 \\
\hline Energy (petroleum) & 50.3 & 53.2 & 73.2 & 84.9 & 68.0 \\
\hline Equipment goods & 99.9 & 92.7 & 112.7 & 106.3 & 114.8 \\
\hline Raw materials and spare parts & 78.0 & 100.1 & 82.2 & 100.0 & 95.3 \\
\hline $\begin{array}{l}\text { Other imports } 1 / \\
\text { ARD }\end{array}$ & \multicolumn{4}{|c|}{ ARD } & 160.9 \\
\hline Food & 3.0 & 3.3 & $\ldots$ & $\ldots$ & $\ldots$ \\
\hline Consumption goods & 17.1 & 18.7 & $\ldots$ & $\ldots$ & $\ldots$ \\
\hline Capital goods & 25.9 & 29.2 & $\ldots$ & $\ldots$ & ... \\
\hline \multicolumn{6}{|l|}{ ZFI } \\
\hline Textiles & 31.4 & 54.8 & $\ldots$ & $\ldots$ & $\ldots$ \\
\hline Capital goods & 4.4 & 7.4 & $\ldots$ & $\ldots$ & $\ldots$ \\
\hline Other & 8.7 & 16.5 & $\cdots$ & $\cdots$ & $\cdots$ \\
\hline Total, c.i.f. & 448.3 & 487.1 & 522.4 & 583.0 & 581.1 \\
\hline \multirow[t]{2}{*}{ Total, f.o.b. } & 381.0 & 414.0 & 444.0 & 495.6 & 493.9 \\
\hline & \multicolumn{5}{|c|}{ (In percent of total imports, c.i.f.) } \\
\hline Food & 12.3 & 9.1 & 7.9 & 6.1 & 7.0 \\
\hline Rice & 5.8 & 2.5 & 1.1 & 2.1 & 2.2 \\
\hline Other & 6.4 & 6.6 & 6.8 & 3.9 & 4.8 \\
\hline Nonfood consumer goods & 16.6 & 14.2 & 14.1 & 15.1 & 17.4 \\
\hline Energy (petroleum) & 11.2 & 10.9 & 14.0 & 14.6 & 11.7 \\
\hline Equipment goods & 22.3 & 19.0 & 21.6 & 18.2 & 19.8 \\
\hline Raw materials and spare parts & 17.4 & 20.5 & 15.7 & 17.1 & 16.4 \\
\hline Other imports $1 /$ & 20.2 & 26.3 & 26.6 & 28.9 & 27.7 \\
\hline \multicolumn{6}{|l|}{ ARD } \\
\hline Food & 0.7 & 0.7 & $\ldots$ & $\ldots$ & ... \\
\hline Consumption goods & 3.8 & 3.8 & ... & $\cdots$ & ... \\
\hline Capital goods & 5.8 & 6.0 & $\ldots$ & $\cdots$ & $\cdots$ \\
\hline \multicolumn{6}{|l|}{$\mathbf{Z F I}$} \\
\hline Textiles & 7.0 & 11.3 & $\ldots$ & ... & $\ldots$ \\
\hline Capital goods & 1.0 & 1.5 & $\ldots$ & $\ldots$ & $\ldots$ \\
\hline Other & 1.9 & 3.4 & $\cdots$ & $\ldots$ & ... \\
\hline Total, c.i.f. & 100.0 & 100.0 & 100.0 & 100.0 & 100.0 \\
\hline
\end{tabular}

Source: Central Bank of Madagascar, and Fund staff estimates.

1/ Includes the imports of the enterprises set up in the cxport processing zone (ZFI) and enterprises under special tax regimes (ARD). 
Table 40. Madagascar: Direction of Trade, 1994-98

(In percent of total)

\begin{tabular}{|c|c|c|c|c|c|}
\hline & 1994 & 1995 & 1996 & 1997 & 1998 \\
\hline Exports, f.o.b. & 100.0 & 100.0 & 100.0 & 100.0 & $\cdots$ \\
\hline European Union & 53.0 & 50.6 & 55.2 & 54.2 & 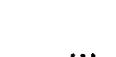 \\
\hline Of which: France & 29.8 & 27.2 & 31.7 & 34.1 & $\cdots$ \\
\hline Other Europe & 0.8 & 4.0 & 2.5 & 3.6 & $\ldots$ \\
\hline United States & 6.9 & 6.3 & 4.2 & 4.7 & $\ldots$ \\
\hline Asia and the Middle East & 10.8 & 7.4 & 20.0 & 11.9 & $\ldots$ \\
\hline Of which: Japan & 7.0 & 5.9 & 15.8 & 3.2 & $\ldots$ \\
\hline Africa & 7.7 & 12.5 & 12.1 & 13.0 & $\ldots$ \\
\hline Of which: Réunion (France) & 5.5 & 5.7 & 5.7 & 5.5 & $\cdots$ \\
\hline Mauritius & 1.7 & 3.7 & 3.9 & 4.9 & $\ldots$ \\
\hline South Africa & 0.4 & $\ldots$ & 0.6 & 1.2 & $\ldots$ \\
\hline Other countries & 20.8 & 19.2 & 6.1 & 12.5 & $\cdots$ \\
\hline Imports, c.i.f. & 100.0 & 100.0 & 100.0 & 100.0 & $\ldots$ \\
\hline European Union & 45.1 & 48.8 & 44.9 & 41.7 & $\ldots$ \\
\hline Of which: France & 29.8 & 30.1 & 30.6 & 26.0 & $\ldots$ \\
\hline Other Europe & 1.7 & 2.2 & 1.1 & 1.1 & $\ldots$ \\
\hline United States & 4.4 & 3.8 & 4.9 & 5.1 & $\ldots$ \\
\hline Asia and the Middle East & 27.9 & 23.2 & 29.4 & 22.7 & $\cdots$ \\
\hline Of which: Iran, Islamic Republic of & 5.0 & 10.2 & 9.0 & 2.2 & $\cdots$ \\
\hline Japan & 9.7 & 6.0 & 5.7 & 7.0 & $\cdots$ \\
\hline Africa & 11.3 & 0.8 & 9.5 & 8.2 & $\ldots$ \\
\hline Of which: Réunion (France) & 0.2 & 0.1 & 0.1 & 0.2 & $\ldots$ \\
\hline Mauritius & 0.5 & 0.5 & 0.9 & 1.0 & $\ldots$ \\
\hline South Africa & 10.2 & $\ldots$ & 8.1 & 6.3 & $\ldots$ \\
\hline Other countries & 9.6 & 21.2 & 10.2 & 21.2 & $\ldots$ \\
\hline
\end{tabular}

Source: Central Bank of Madagascar, Bulletin d'Information et de Statistiques, Supplément Annuel, 1997. 
Table 41. Madagascar: Balance of Services, 1994-98 (In millions of SDRs)

\begin{tabular}{|c|c|c|c|c|c|}
\hline & 1994 & 1995 & 1996 & $\begin{array}{r}1997 \\
\text { Est. }\end{array}$ & $\begin{array}{l}1998 \\
\text { Prov. }\end{array}$ \\
\hline Credit & 145.1 & 162.1 & 210.7 & 200.3 & 211.6 \\
\hline Freight and insurance on goods & 3.0 & 3.3 & 3.3 & 3.3 & 3.5 \\
\hline Other transport & 44.1 & 44.2 & 52.2 & 40.4 & 38.2 \\
\hline Travel & 32.2 & 38.0 & 44.6 & 52.9 & 63.5 \\
\hline Investment income & 1.7 & 4.6 & 5.9 & 5.7 & 13.2 \\
\hline Other services, public & 16.4 & 16.0 & 28.3 & 20.9 & 20.9 \\
\hline Other services, private & 47.7 & 56.0 & 76.4 & 77.1 & 72.3 \\
\hline Debit & 335.0 & 355.5 & 382.2 & 357.6 & 365.5 \\
\hline Freight and insurance on goods & 61.2 & 67.5 & 72.4 & 79.2 & 78.4 \\
\hline Percent of imports, c.i.f. & 13.7 & 13.9 & 13.9 & 13.6 & 13.5 \\
\hline Other transport & 36.1 & 37.6 & 37.2 & 34.3 & 24.3 \\
\hline Travel & 32.6 & 39.7 & 49.7 & 56.9 & 83.9 \\
\hline Investment income & 105.6 & 114.5 & 114.9 & 80.8 & 60.5 \\
\hline Interest & 104.7 & 112.3 & 112.9 & 75.8 & 55.5 \\
\hline Dividends & 0.9 & 2.2 & 2.0 & 5.0 & 5.0 \\
\hline Other services: public & 55.2 & 53.6 & 64.5 & 69.4 & 82.0 \\
\hline Government expenditures & 8.4 & 10.2 & 10.3 & 10.7 & 14.4 \\
\hline Services linked to project loans & 36.7 & 25.9 & 32.8 & 33.5 & 40.9 \\
\hline Services linked to project grants & 10.1 & 17.5 & 21.4 & 25.2 & 26.7 \\
\hline Other services: private & 44.3 & 42.6 & 43.5 & 37.0 & 36.4 \\
\hline Net services & -189.9 & -193.4 & -171.6 & -157.3 & -153.9 \\
\hline Nonfactor services & -86.0 & -83.5 & -62.5 & -82.2 & -106.6 \\
\hline Factor services & -103.9 & -109.9 & -109.0 & -75.1 & -47.3 \\
\hline
\end{tabular}

Sources: Central Bank of Madagascar; and Fund staff estimates. 
Table 42. Madagascar: Stock of External Debt, 1994-98

(In millions of SDRs)

\begin{tabular}{|c|c|c|c|c|c|}
\hline & 1994 & 1995 & 1996 & 1997 & $\begin{array}{r}1998 \\
\text { Est. }\end{array}$ \\
\hline Medium and long-term official debt & $1,721.2$ & $1,635.2$ & $1,603.2$ & $1,604.8$ & $1,767.5$ \\
\hline Official creditors & 668.4 & 562.3 & 483.5 & 425.3 & 383.9 \\
\hline Paris Club & 574.8 & 510.1 & 463.7 & 404.8 & 363.4 \\
\hline Other countries & 93.6 & 52.2 & 19.9 & 20.5 & 20.5 \\
\hline International organizations & $1,052.8$ & $1,072.8$ & $1,119.7$ & $1,179.4$ & $1,383.7$ \\
\hline $\mathbb{M M F}$ & 59.2 & 48.9 & 50.8 & 51.5 & 41.3 \\
\hline Others & 993.6 & $1,024.0$ & $1,068.8$ & $1,127.9$ & $1,342.4$ \\
\hline Of which: IBRD/IDA & 606.2 & 643.1 & 785.5 & 800.5 & 927.3 \\
\hline African Development Bank & 40.8 & 35.2 & 26.3 & 27.5 & 28.4 \\
\hline African Development Fund & 134.1 & 131.2 & 131.7 & 140.2 & 225.0 \\
\hline European Investment Bank & 59.8 & 62.5 & 52.4 & 57.1 & 48.9 \\
\hline Private sector & 27.0 & 17.7 & 12.8 & 5.0 & 3.6 \\
\hline Commercial banks & 19.9 & 13.6 & 5.9 & 0.7 & 0.5 \\
\hline Other private creditors & 7.0 & 4.1 & 6.9 & 4.3 & 3.1 \\
\hline Other debt & $1,094.0$ & $1,280.3$ & $1,469.1$ & $1,494.9$ & $1,167.3$ \\
\hline Of which: Short term & 15.4 & 0.0 & 0.0 & 0.0 & 0.0 \\
\hline Total arrears & $1,078.6$ & $1,280.3$ & $1,469.1$ & 717.0 & 364.8 \\
\hline Paris Club & 523.0 & 680.5 & $1,130.4$ & 387.4 & 0.0 \\
\hline Other bilateral creditors & 523.3 & 555.3 & 268.6 & 301.2 & 332.5 \\
\hline Multilaterals & 15.5 & 31.9 & 41.8 & 1.0 & 0.0 \\
\hline Private sector & 16.8 & 19.2 & 28.3 & 30.7 & 32.2 \\
\hline Total external debt outstanding $1 /$ & $2,842.2$ & $2,933.2$ & $3,085.1$ & $3,104.7$ & $2,938.5$ \\
\hline \multicolumn{6}{|l|}{ Memorandum items: } \\
\hline Total external debt before debt relief & $2,842.2$ & $2,933.2$ & $3,085.1$ & $3,178.3$ & $3,322.5$ \\
\hline \multicolumn{6}{|l|}{ Total external debt /GDP (in percent) } \\
\hline Before debt relief & 136.6 & 140.9 & 111.9 & 123.5 & 121.2 \\
\hline After debt relief & 136.6 & 140.9 & 111.9 & 120.6 & 107.2 \\
\hline Total external debt (before debt relief) $2 /$ & 620.2 & 584.2 & 545.7 & 566.2 & 569.3 \\
\hline Late interest payments & 43.2 & 58.3 & 70.0 & 23.5 & 8.4 \\
\hline Charges on rescheduling & 0.0 & 0.0 & 0.0 & 15.6 & 13.7 \\
\hline Total debt to official creditors & $2,798.4$ & $2,902.9$ & $3,044.0$ & $2,294.3$ & $2,100.1$ \\
\hline
\end{tabular}

Sources: Central Bank of Madagascar; and Fund staff estimates.

1/ After debt relief.

$2 /$ In percent of exports of goods and nonfactor services. 
Figure 1. Madagascar: Selected Economic Indicators, 1994-1998
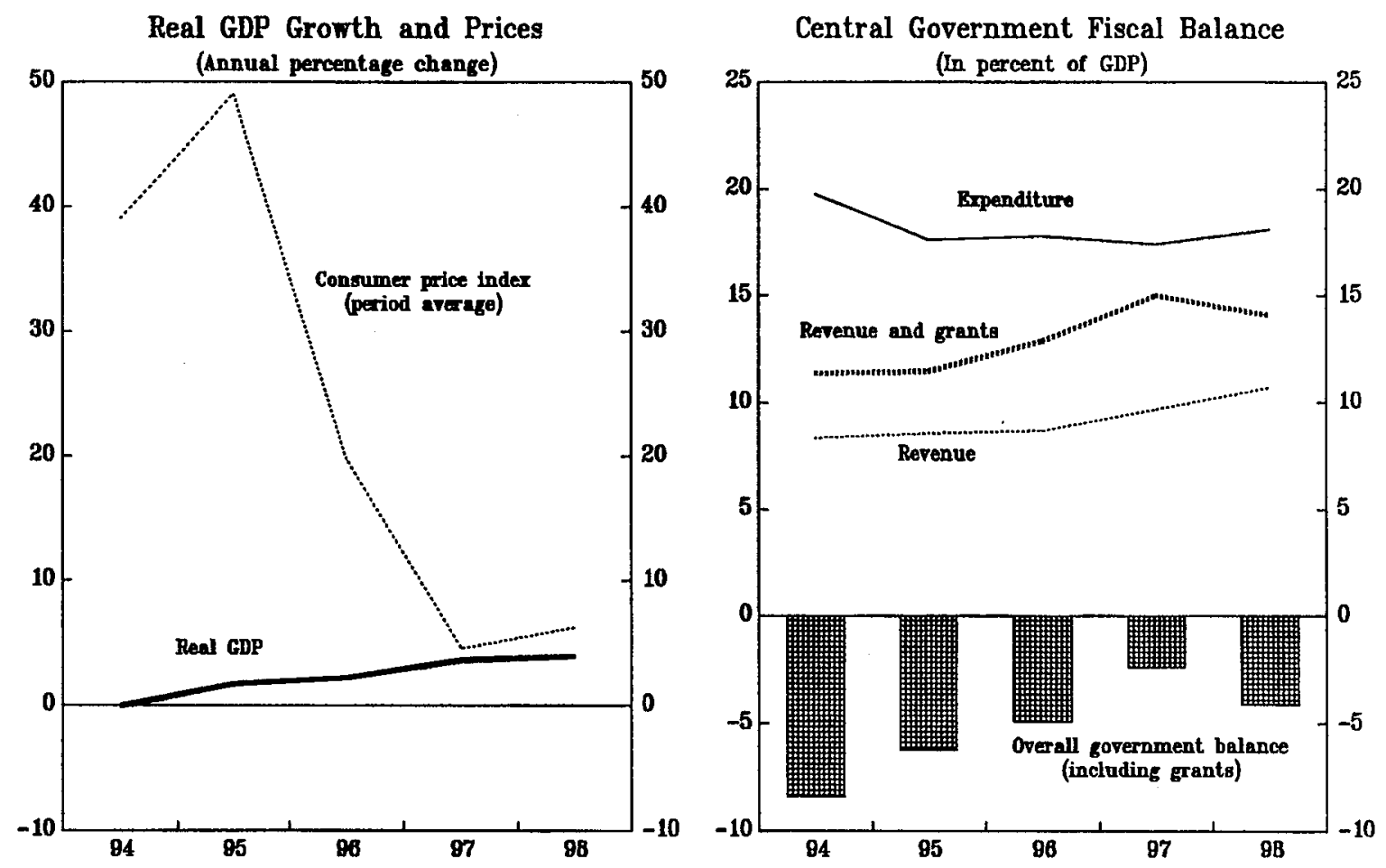

External Current Account

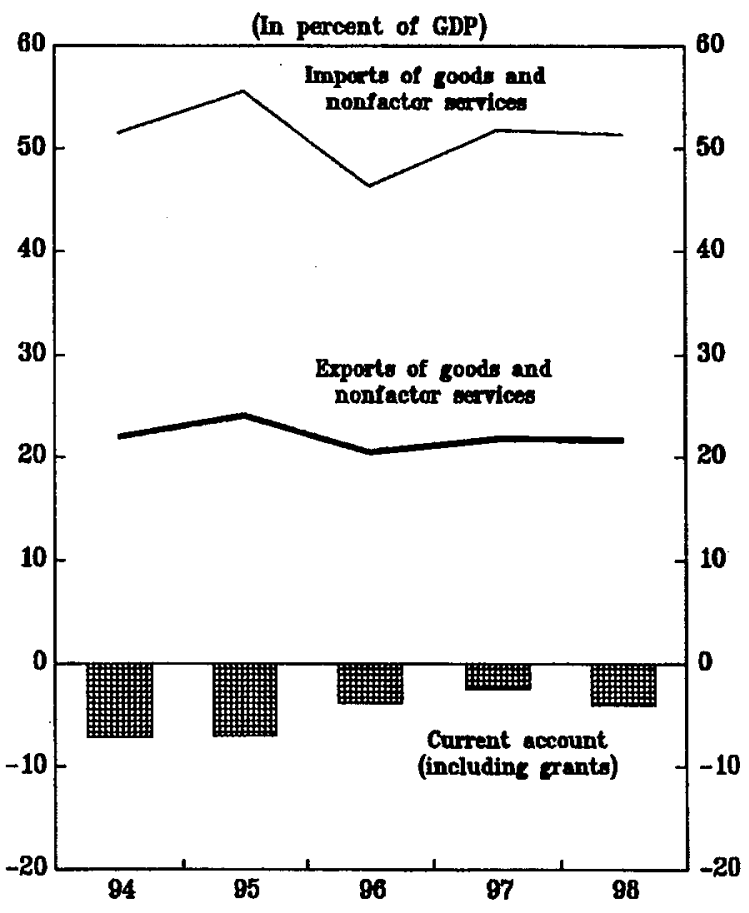

Saving and Investment

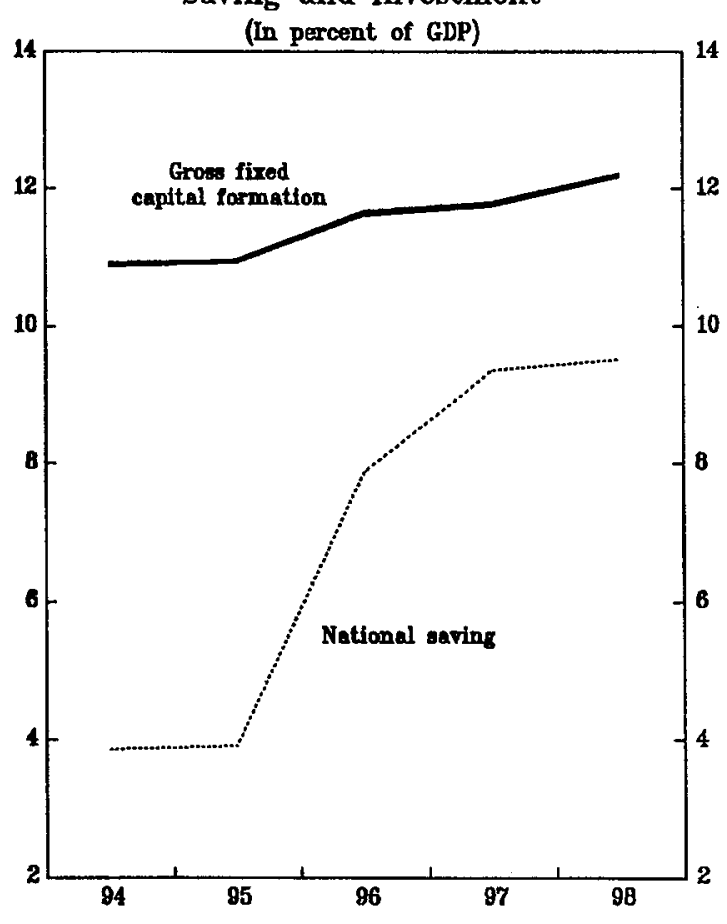

Sources: IMF, Forld Economic Outlook database; and Fund staff estimates and projections. 
Figure 2. Madagascar: Selected Exchange Rate Indices, 1990-98

(1990=100; foreign currency per Malagasy franc)

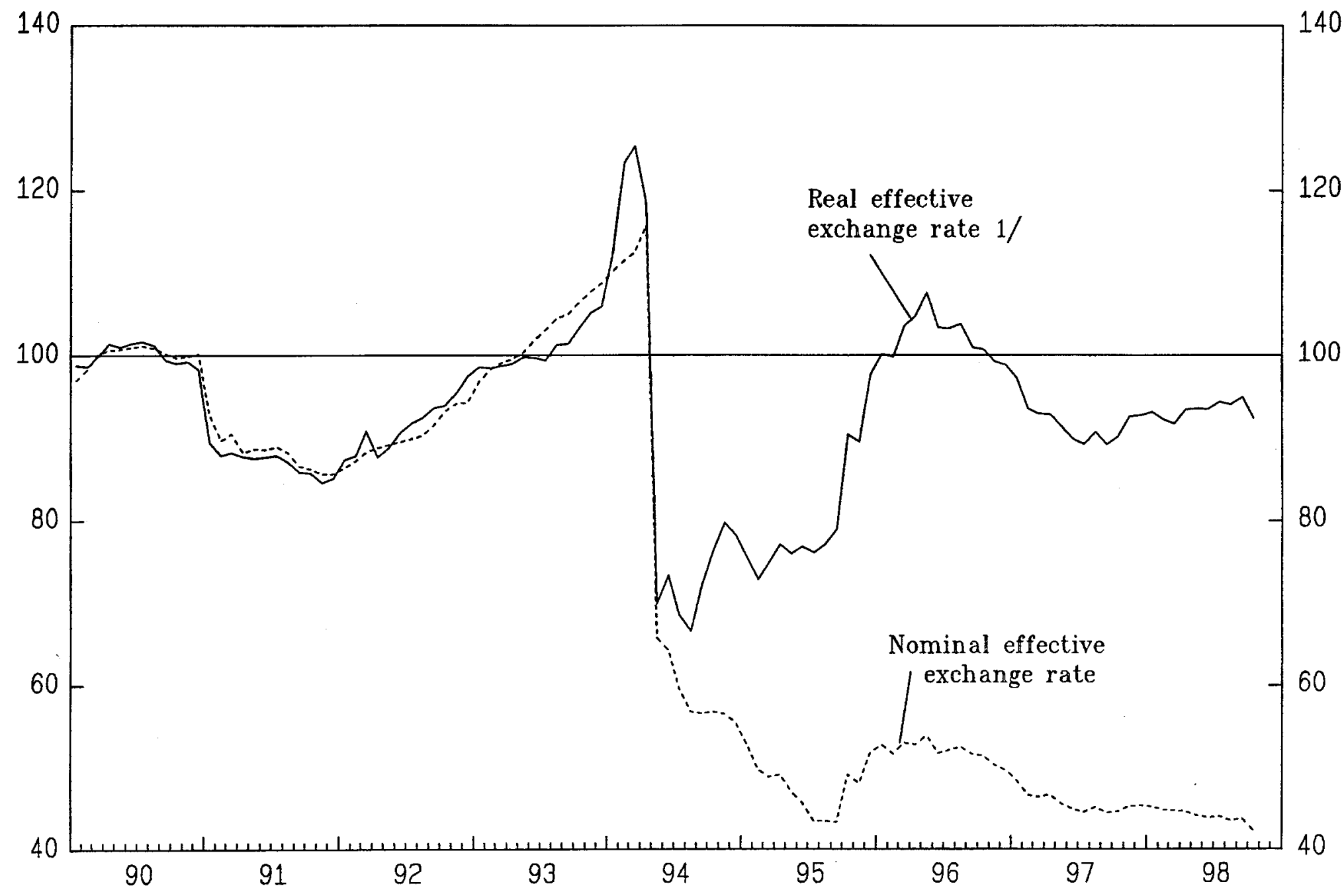

Source: IMF, Information Notice System.

1/ Trade-reighted index of nominal exchange rate deflated by seasonally adjusted relative consumer prices; an increase (decrease) indicates appreciation (depreciation). 
Figure 3. Madagascar : Interest Rates, 1995-98

(In percent per annum)

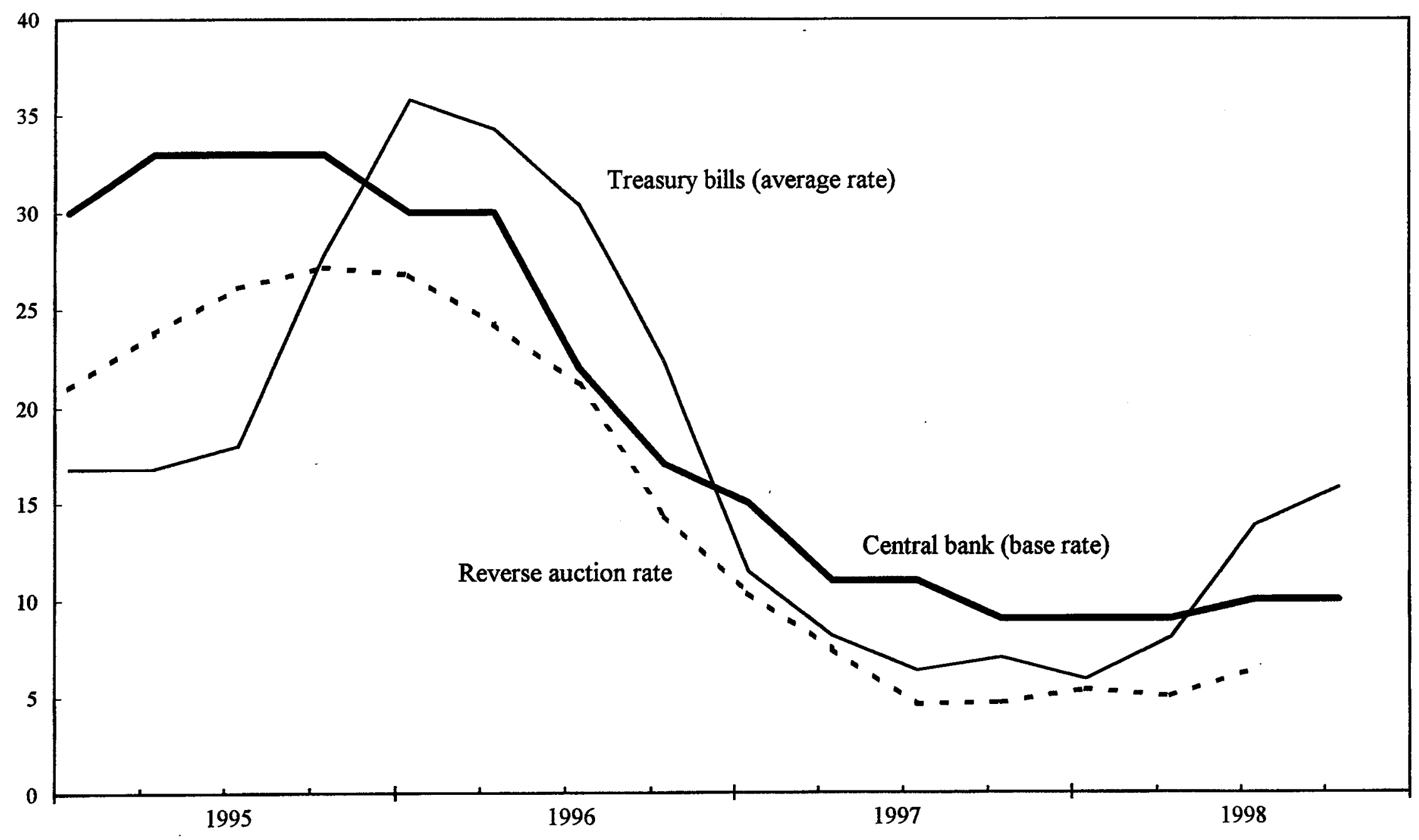


Figure 4. Madagascar : Commercial Banks' Interest Rates and Inflation, 1995-98

(In percent per annum)

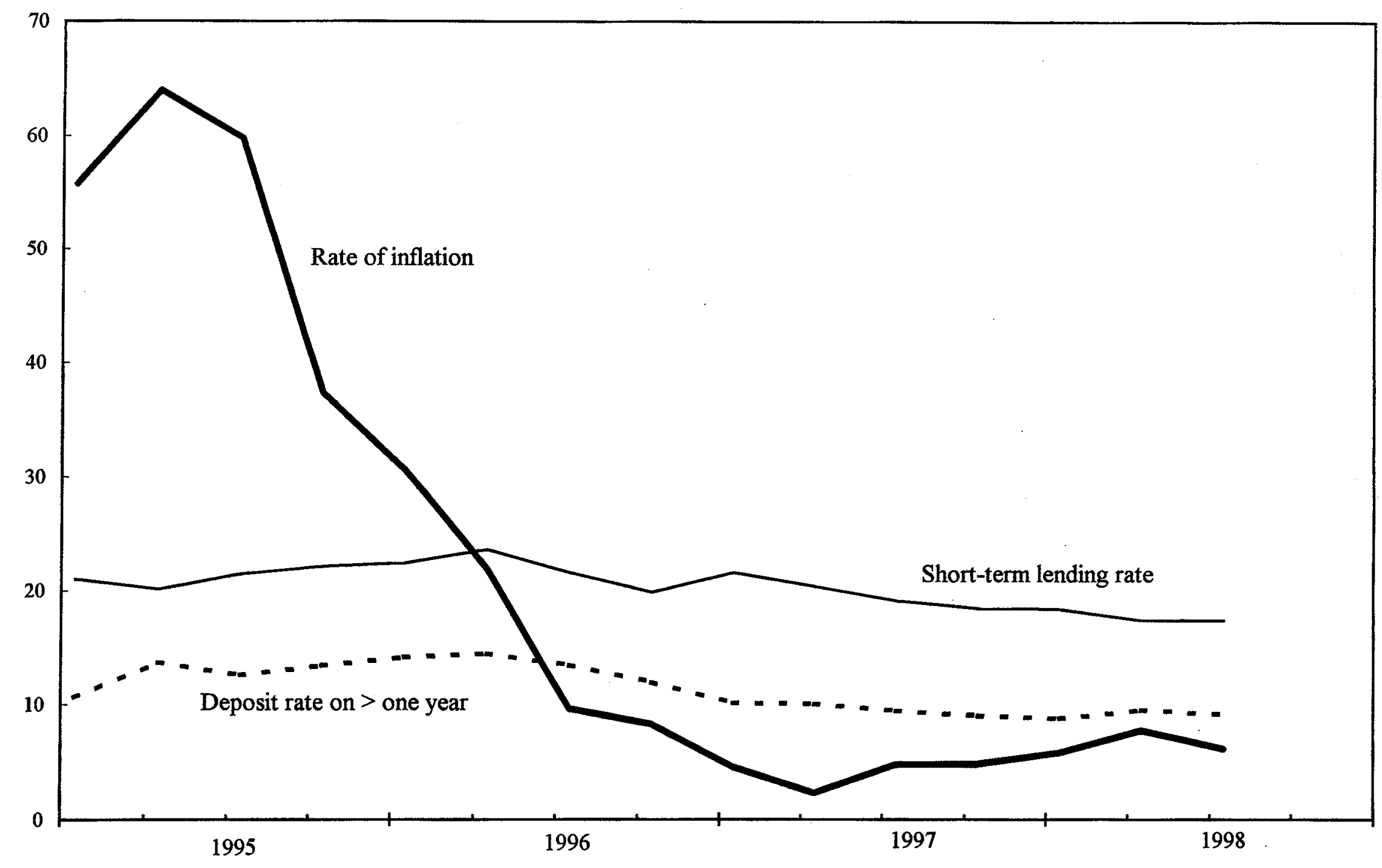


Figure 5. Madagascar : Bank Liquidity, 1995-98

(In percent of required reserves)

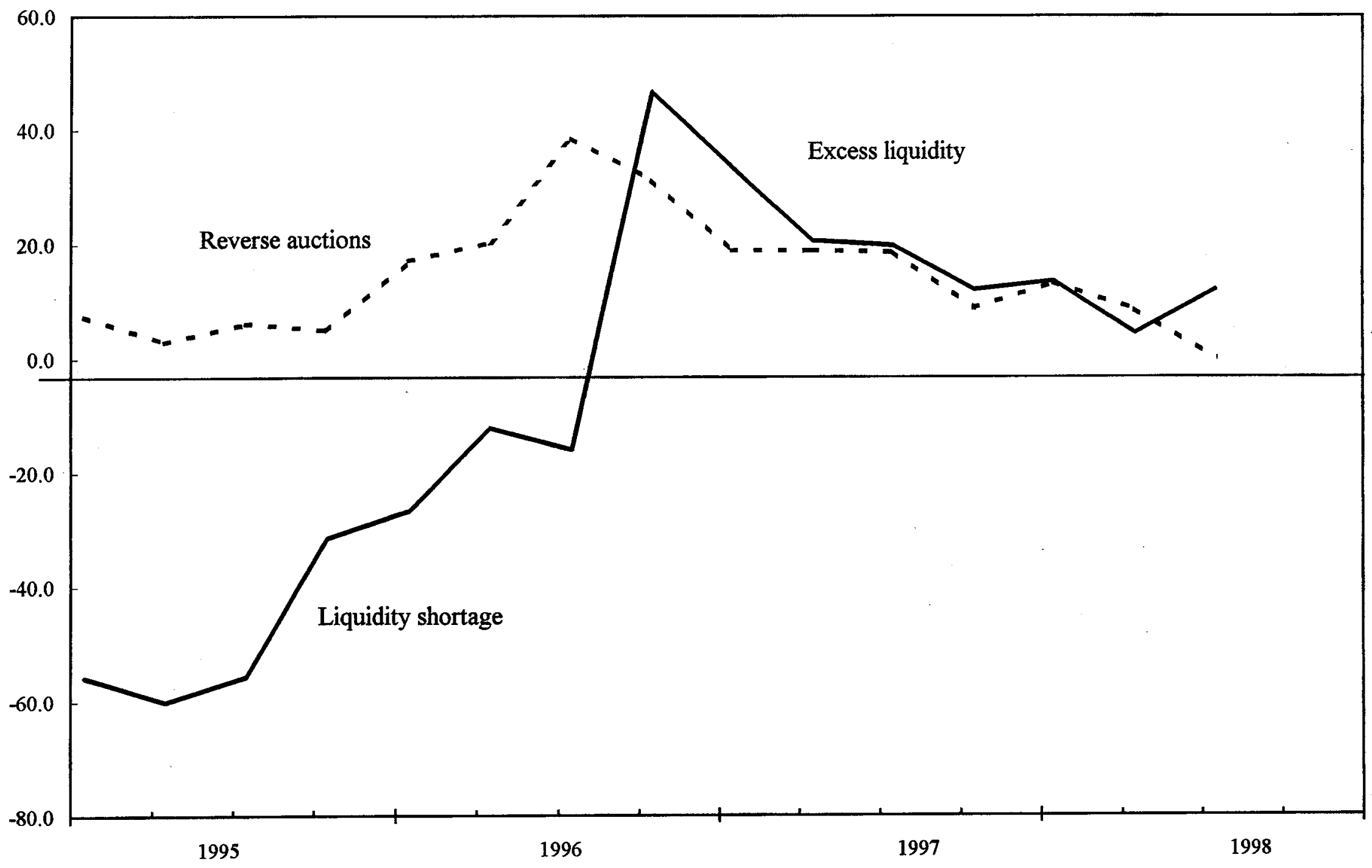

